\title{
New insights into molecular pathways associated with flatfish ovarian development and atresia revealed by transcriptional analysis
}

\author{
Angèle Tingaud-Sequeira1,2, François Chauvigné ${ }^{1}$, Juanjo Lozano ${ }^{3}$, \\ María J Agulleiro ${ }^{1,4}$, Esther Asensio ${ }^{5}$ and Joan Cerdà*1
}

Address: ${ }^{1}$ Laboratory of Institut de Recerca i Tecnologia Agroalimentàries (IRTA)-Institut de Ciències del Mar, Consejo Superior de Investigaciones Científicas (CSIC), 08003 Barcelona, Spain, ${ }^{2}$ Génomique et Physiologie des Poissons, Université Bordeaux 1, UMR NuAGe, 33405 Talence, France, ${ }^{3}$ Centro de Investigación Biomédica en Red de Enfermedades Hepáticas y Digestivas (CIBERehd), 08036 Barcelona, Spain, ${ }^{4}$ Instituto de Acuicultura Torre de la Sal (CSIC), 12595 Castellón, Spain and 5IFAPA Centro El Toruño, Junta de Andalucía, Cádiz, Spain

Email: Angèle Tingaud-Sequeira - angela.tingaud@u-bordeaux1.fr; François Chauvigné - chauvigne@cmima.csic.es; Juanjo Lozano - juanjo.lozano@ciberehd.org; María J Agulleiro - mjagulleiro@iats.csic.es; Esther Asensio - esther.asensio.ext@juntadeandalucia.es; Joan Cerdà* - joan.cerda@irta.es

* Corresponding author

Published: 15 September 2009

BMC Genomics 2009, 10:434 doi:10.1 |86/|47|-2/64-10-434
Received: II May 2009

Accepted: 15 September 2009

This article is available from: http://www.biomedcentral.com//47/-2/64//10/434

(C) 2009 Tingaud-Sequeira et al; licensee BioMed Central Ltd.

This is an Open Access article distributed under the terms of the Creative Commons Attribution License (http://creativecommons.org/licenses/by/2.0), which permits unrestricted use, distribution, and reproduction in any medium, provided the original work is properly cited.

\begin{abstract}
Background: The Senegalese sole (Solea senegalensis) is a marine flatfish of increasing commercial interest. However, the reproduction of this species in captivity is not yet controlled mainly because of the poor knowledge on its reproductive physiology, as it occurs for other non-salmonid marine teleosts that exhibit group-synchronous ovarian follicle development. In order to investigate intra-ovarian molecular mechanisms in Senegalese sole, the aim of the present study was to identify differentially expressed genes in the ovary during oocyte growth (vitellogenesis), maturation and ovarian follicle atresia using a recently developed oligonucleotide microarray.

Results: Microarray analysis led to the identification of 118 differentially expressed transcripts, of which 20 and 8 were monitored by real-time PCR and in situ hybridization, respectively. During vitellogenesis, many up-regulated ovarian transcripts had putative mitochondrial function/location suggesting high energy production (NADH dehydrogenase subunits, cytochromes) and increased antioxidant protection (selenoprotein W2a), whereas other regulated transcripts were related to cytoskeleton and zona radiata organization (zona glycoprotein 3, alpha and beta actin, keratin 8), intracellular signalling pathways (heat shock protein 90, Ras homolog member G), cell-to-cell and cell-to-matrix interactions (beta I integrin, thrombospondin 4b), and the maternal RNA pool (transducer of ERBB2 Ia, neurexin la). Transcripts up-regulated in the ovary during oocyte maturation included ion transporters $\left(\mathrm{Na}^{+}-\mathrm{K}^{+}-\mathrm{ATPase}\right.$ subunits), probably required for oocyte hydration, as well as a proteinase inhibitor (alpha-2-macroglobulin) and a vesicle calcium sensor protein (extended synaptotagmin-2-A). During follicular atresia, few transcripts were found to be up-regulated, but remarkably most of them were localized in follicular cells of atretic follicles, and they had inferred roles in lipid transport (apolipoprotein C-I), chemotaxis (leukocyte cell-derived chemotaxin 2,), angiogenesis (thrombospondin), and prevention of apoptosis (SI00a IO calcium binding protein).
\end{abstract}

Conclusion: This study has identified a number of differentially expressed genes in the ovary that were not previously found to be regulated during ovarian development in marine fish. Specifically, we found evidence, for the first time in teleosts, of the activation of chemoattractant, angiogenic and antiapoptotic pathways in hypertrophied follicular cells at the onset of ovarian atresia. 


\section{Background}

Our understanding of the molecular pathways underlying reproductive processes and oogenesis in vertebrates is still limited even in mammalian models. Teleosts show the most diversified reproductive strategies among vertebrates making it even more difficult to uncover the underlying molecular mechanisms. In addition, several factors can modulate the reproductive processes of teleost fish such as photoperiod and temperature [1,2], nutrition [3], captivity [4] and endocrine disruptors [5,6]. Accordingly, most studies to date on female teleosts have mainly investigated the effect of these conditions on the circulating sex hormone levels or the reproductive success in terms of spawning performance (e.g., fecundity, egg and larval survival). However, the molecular and cellular mechanisms involved still remain poorly understood.

The development of methods for large-scale gene expression analysis (e.g., microarrays) in model fish species, such as the zebrafish (Danio rerio), as well as in salmonids is improving our knowledge of the molecular basis of ovarian physiology in teleosts [7]. In the zebrafish, cDNAand oligo-based microarrays have been employed to assess the transcriptome profile of differentiating and adult gonads. These studies have identified a number of genes involved in mitochondrial organization and biogenesis, cell growth and maintenance, and germ-line differentiation, as well as some with sexually dimorphic coexpression in both the gonads and the brains [8-10]. Mass sequencing of zebrafish expressed sequence tags (ESTs) from the ovary [11], or from isolated fully-grown ovarian follicles through serial analysis of gene expression (SAGE) [12], has also discovered germ cell-specific genes and established the complete sequence data set of maternal mRNA stored in oocytes at the end of oogenesis. In rainbow trout (Oncorhynchus mykiss) and coho salmon (O. kisutch), cDNA microarrays printed on slides or nylon membranes, as well as reciprocal suppression subtractive hybridization ( $\mathrm{SSH}$ ), were used to investigate changes in the ovarian transcriptome during primary growth and maturation and bacterial lipopolysaccharide-induced ovarian apoptosis [13-17]. These studies revealed changes in the expression of genes involved in cellular organization and extracellular matrix (ECM) remodelling, immunoregulation, apoptosis, cell cycling, and in different endocrine and paracrine systems, which might be important during ovarian development. The control of ovulation by either hormonal induction or photoperiod manipulation has also been shown to induce differences in the egg mRNA abundance of specific genes, which may affect their developmental competence [18].

However, despite the significant information obtained from zebrafish and salmonids, no data is currently available on the changes of the transcriptome during ovarian development in other marine teleosts, such as flatfish, some of which are of economical importance. Particularly, little is known on the molecular pathways involved in ovarian follicular atresia, a degenerative and resorptive process of ovarian follicles that determines fecundity in both natural and captive conditions [19-22]. The use of functional genomics approaches would contribute with the identification of molecular signatures associated with abnormal ovarian development or premature ovarian regression in cultured fish species, thus providing potentially useful markers to control sexual maturation.

The Senegalese sole, Solea senegalensis, is a marine flatfish of high commercial value in Southern Europe and Asia [23]. However, the industrial production of this species is largely based on wild breeders after long periods of acclimation to captivity, and reproduction is not yet controlled [26]. The F1 generation of fish raised in captivity often fail to reproduce naturally because egg fertilization is dramatically reduced [24,25]. In some F1 females, an increased ovarian follicle atresia and/or dysfunctions of the ovulatory process might also occur, but no precise studies have been performed to clarify this phenomenon. In order to obtain information on the molecular basis of ovarian development in Senegalese sole, the present study aimed at performing a transcriptomic analysis of the ovary during oocyte growth (vitellogenesis), maturation and follicular atresia using a recently developed oligonucleotide microarray [27]. The analysis revealed the differential expression of more than one hundred genes during ovarian development, some of them with yet unknown functions in the fish ovary. In addition, determination of the cell type-specific expression in the ovary of selected transcripts suggest the activation of genes presumably involved in chemotaxis, angiogenesis and prevention of apoptosis in follicular cells of atretic follicles, which have not been described before in teleosts during ovarian atresia.

\section{Results}

\section{Stages of ovarian development}

To identify differentially expressed genes during ovarian development in Senegalese sole, samples of ovarian tissue were collected from adult females sacrificed throughout the annual reproductive cycle $[28,29]$ or after hormonal treatment (Figure 1). Thus, samples of ovaries at previtellogenesis (Figure 1A and 1B), vitellogenesis (Figure 1D and $1 \mathrm{E}$ ), maturation (Figure $1 \mathrm{G}$ and $1 \mathrm{H}$ ), and undergoing follicular atresia (Figure 1J, K and 1L), were used for transcriptome analysis. As many other fractional spawner teleosts, the Senegalese sole has a group-synchronous ovary in which follicles of all sizes up through vitellogenesis are present at any time, and populations (or clutches) of follicles are periodically recruited into maturation from a population of oocytes in late vitellogenic stages [30]. 

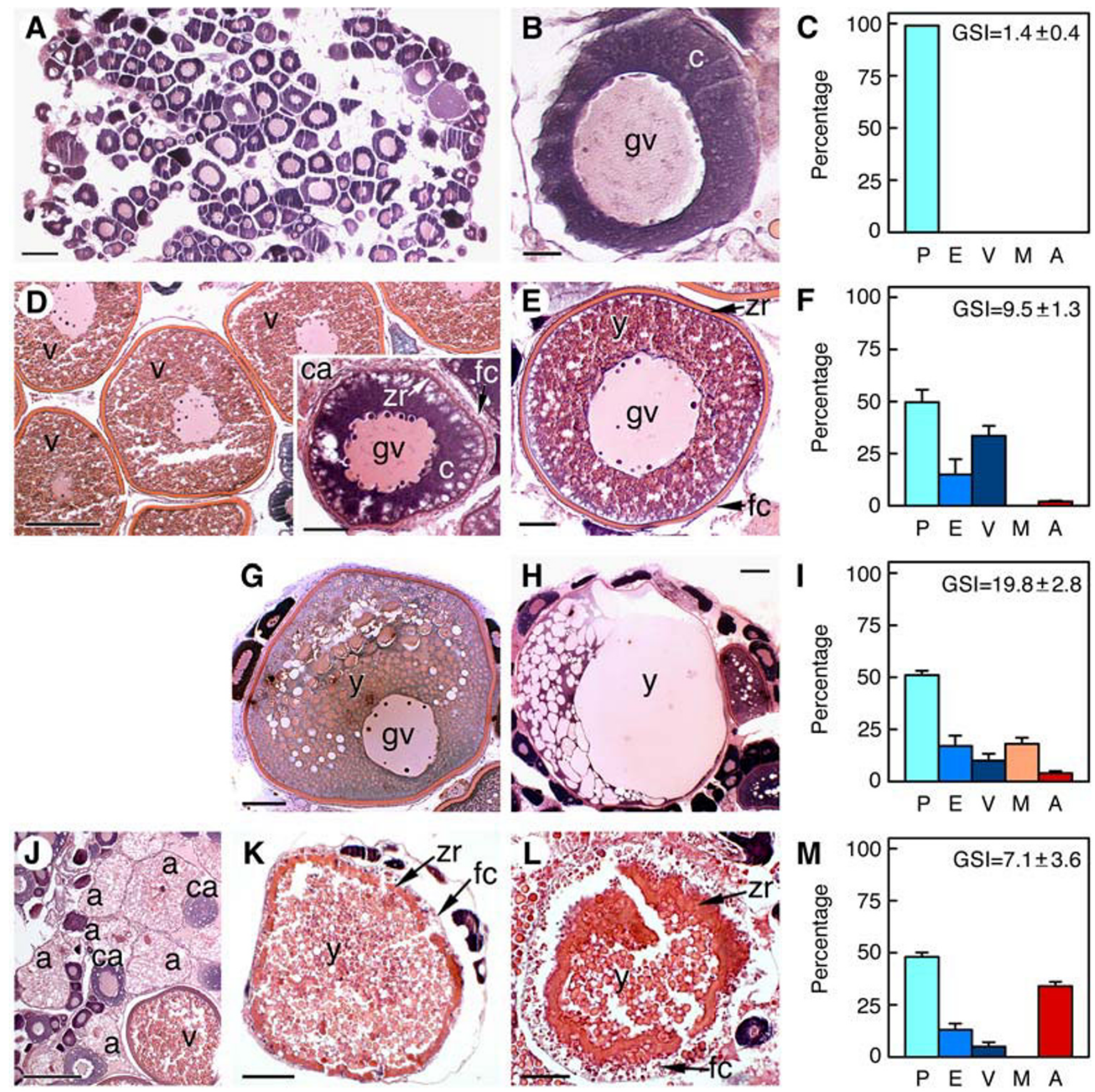

\section{Figure I}

Developmental stage of the Senegalese sole ovaries used for microarray analysis. Representative light micrographs of histological sections of the ovary ( $n=3$ females) stained with hematoxylin-eosin $(A, B, D, E, G, H$, and J-L), and frequency of ovarian follicles in the ovary $(C, F, I$ and $M)$ at each ovarian developmental stage, previtellogenic $(A$ and $B)$, vitellogenic (D and $E)$, mature $(G$ and $H)$ and atretic $(J-L)$. Data on the frequency of ovarian follicles are the mean \pm SEM $(n=3$ females). (B) Oocyte at primary growth stage. (D inset) Ovarian follicle containing a cortical alveolus stage oocyte. (G) Follicle at early maturation (note the migration of the germinal vesicle). $(\mathrm{H})$ Follicle containing a mature oocyte (the germinal vesicle is not observed and yolk is fused). (K) Ovarian follicle at early atresia. (L) Follicle at advanced atresia. gv, germinal vesicle; c, oocyte cytoplasm; y, yolk; zr, zona radiata; fc, follicular cells; ca, cortical alveolus stage oocyte. P, previtellogenic follicle; E, early vitellogenic follicle; $V$, vitellogenic follicle; $M$, mature follicle; $A$, atretic follicle. Bars, $200 \mu \mathrm{m}(D, J), I 00 \mu \mathrm{m}(A, G, H, K), 50 \mu \mathrm{m}(E$, $\mathrm{D}$ inset, L), $20 \mu \mathrm{m}(\mathrm{B})$. 
Therefore, the increased frequency of vitellogenic, mature or atretic ovarian follicles in the ovary, as determined by histological analysis (Figure 1C, F, I and 1M), defined the ovarian developmental stages used in the present study.

The previtellogenic ovary was formed by exclusively ovarian follicles with oocytes at the primary growth stage (oocyte/follicle diameter up to approximately $150 \mu \mathrm{m}$ ) in which vitellogenin incorporation and yolk formation did not yet start (Figure 1A-1C). In the vitellogenic ovary, a population of follicles were recruited into vitellogenesis, and consequently the proportion of follicles at the primary growth stage decreased (Figure 1D-1F). At this stage, follicles containing oocytes at the cortical alveolus stage (up to approximately $300 \mu \mathrm{m}$ ), characterized by the presence of nascent cortical alveoli within the ooplasm, were more abundant (Figure 1D, inset). Vitellogenic oocytes surrounded by the zona radiata and the somatic follicular cells, granulosa and theca cells, increased in size (up to $500 \mu \mathrm{m}$ in diameter at late vitellogenesis) and their cytoplasm was filled with yolk granules where vitellogeninderived yolk proteins are stored (Figure 1E). As a result of this growing phase, the gonadosomatic index (GSI) of females increased by approximately 7 -fold (Figure 1C and $1 \mathrm{~F})$.

Maturing ovaries containing follicle-enclosed oocytes undergoing meiosis resumption, and ovaries carrying mature oocytes prior to ovulation, were collected 24-48 h after treatment of vitellogenic females with gonadotropinreleasing hormone agonist [D-Ala $\left.{ }^{6}, \mathrm{Pro}^{9}, \mathrm{NEt}\right]$ (GnRHa) [24]. In the mature ovary, a population of follicleenclosed oocytes at late stages of vitellogenesis was further recruited into maturation (Figure 1G-1I). In these oocytes, the germinal vesicle migrates towards the animal pole and yolk globules fuse one another (Figure 1G), eventually forming a large mass of yolk (Figure $1 \mathrm{H}$ ). The mature oocyte reached $800-900 \mu \mathrm{m}$ in diameter due to water uptake (hydration), resulting in a further 2-fold increase of the GSI (Figure 1F and 1I).

Finally, atretic ovaries were collected from females showing spontaneously occurring ovarian follicle atresia during the spawning season, or induced after GnRHa treatment. In these ovaries, approximately up to $30-40 \%$ of the ovarian follicles showed different levels of atresia and maturing/mature oocytes were absent (Figure 1J-1M). In early atretic follicles, vitellogenic oocytes shrank, the zona radiata folded, and follicles became irregularly shaped (Figure $1 \mathrm{~K}$ and $1 \mathrm{~L}$ ). The follicular cells were hypertrophied and the theca was poorly developed. Advanced follicular atresia was characterized by breakdown and resorption of the zona radiata, and the appearance of highly columnar follicular cells apparently showing an intense phagocytic activity as suggested by the presence of large vacuoles (Figure 2). At this stage, accumulation of blood cells, erythrocytes and leukocytes in the follicle, as well as in the oocyte, was also noted (Figure 2B).

\section{Microarray analysis}

Differential gene expression in the four ovarian developmental stages was determined using a Senegalese sole-specific oligonucleotide microarray containing 60-mer probes representing 5,087 unique genes [27]. This platform was previously designed from a Senegalese sole EST database derived from a multi-tissue normalized cDNA library from different adult tissues (including ovaries at
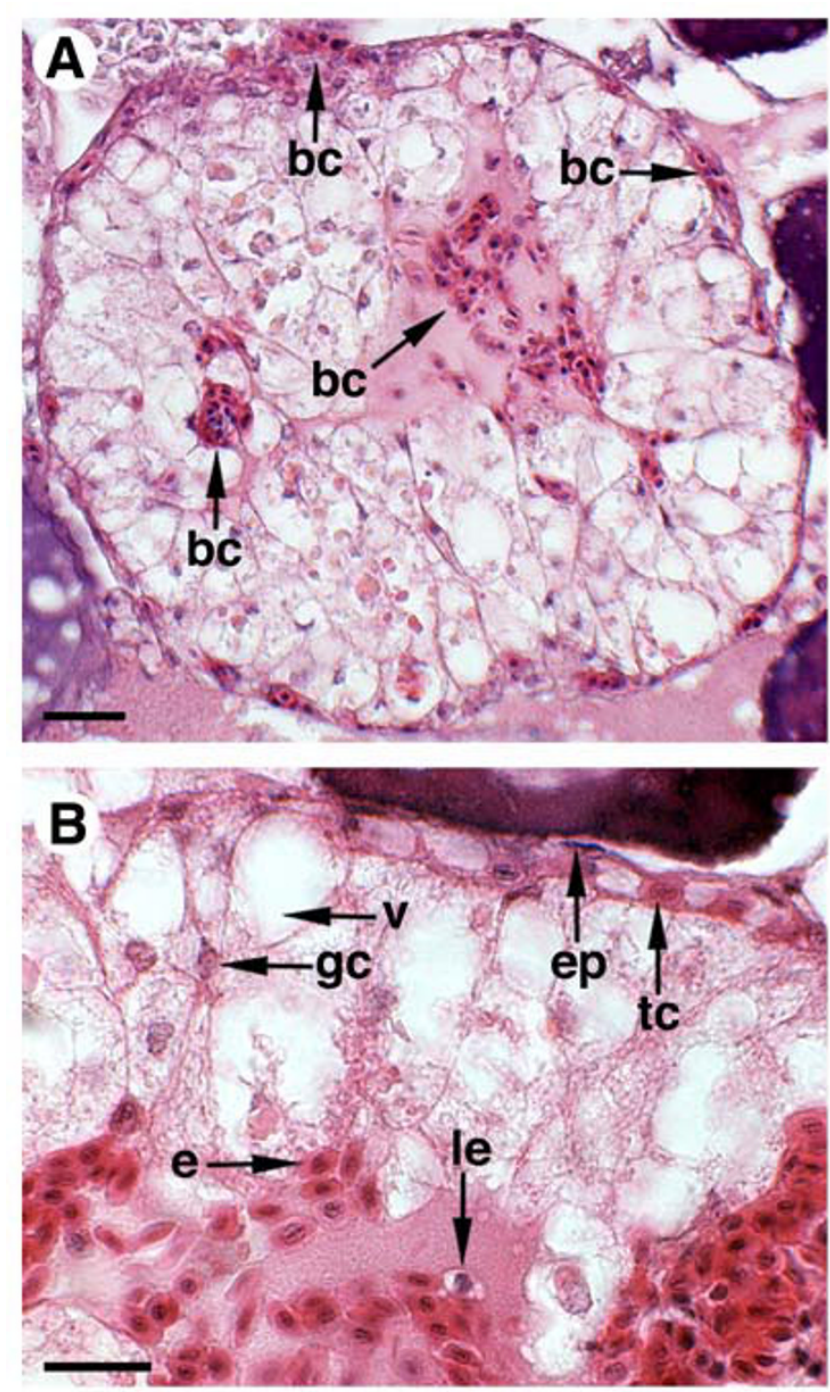

Figure 2

Photomicrographs of ovarian follicles at advanced atresia. Light micrographs of histological sections stained with hematoxylin-eosin. bc, blood cells; ep, epithelium; tc, theca cells; v, vacuole; gc, granulosa cells; e, erythrocytes; le, leukocytes. Bars, $20 \mu \mathrm{m}$. 
different developmental stage) and larval and juvenile stages [27]. Therefore, although this platform was not ovary-specific and most likely did not contain all the transcripts expressed in the sole ovary, its was useful to obtain a first insight into the overall changes of gene expression during ovarian development.

To determine the false discovery rate (FDR) in each of the differential gene expression experiments (vitellogenic $v$ s. previtellogenic ovaries, mature vs. vitellogenic ovaries, and atretic $v s$. vitellogenic/mature ovaries), an additional microarray experiment was performed by hybridizing differentially labelled (Cy3 and Cy5) aliquots of amplified RNA (aRNA) from the same sample (previtellogenic ovary). As expected, there were few differences between the Cy3 and Cy5 signals for most of the microarray spots in these experiments giving an estimated overall FDR of $3.0,3.8$ and $5.6 \%$ for vitellogenic $v$ s. previtellogenic ovaries, mature $v s$. vitellogenic ovaries, and atretic $v s$. vitellogenic/mature ovaries, respectively (see Additional file 1).

Microarray data analysis indicated significant $(p<0.01)$ regulation of genes in vitellogenic (46 ESTs), mature (46 ESTs) and atretic ovaries (26 ESTs), which showed fold change (FC) values from 1.4 up to 5.1. These ESTs, and the corresponding GenBank accession numbers are listed in Tables 1, 2 and 3. In Table 3, differential expressed genes in atretic ovaries relative to vitellogenic or mature ovaries are pooled together. Some of these ESTs (26\%) could not be annotated, even after sequencing the respective clones from the 5 ' end, and are not included in these tables.

\section{Gene ontology annotation}

To obtain a first assessment of the more important physiological processes occurring during ovarian development, gene ontology (GO) analysis was carried out using the BLAST2GO v1 program [31]. Most of the annotated ESTs (93\%) had GO assignments, and many of those had 3-6 assignments each (49\%) and a significant proportion (34\%) had 7 or more assignments.

Figure 3 shows the differentially expressed genes in the three ovarian stages (vitellogenesis, maturation and follicular atresia) classified according to GO terms biological process (level 3), cellular component (level 5) and molecular function (level 3). During vitellogenesis, the majority of regulated ESTs were dedicated to metabolic process, oxidation reduction, regulation and anatomical structure development, in the biological process category. A similar distribution of GO terms was seen within the EST cluster regulated during maturation, although in this case transcripts related to cell cycle, localization of cell, cellular component organization, and system process, were also detected. During ovarian follicle atresia, most regulated genes fall in the cellular metabolism, establishment of localization, and cellular component organization attributes.

During vitellogenesis and maturation, most protein products were mainly inferred to be associated with mitochondria based on the cellular component category, although some also might be in the cytoskeleton (specially during vitellogenesis), the nucleus, and intracellularly in organelles. Interestingly, during atresia, the products of most of the up-regulated genes showed putative extracellular location, whereas the products of the down-regulated genes had membrane and nucleus locations.

Finally, classification using the molecular function category indicated that most of the gene products regulated during vitellogenesis and maturation were dedicated to binding and catalytic functions, including nucleotide binding, protein binding, ion binding, and transferase and hydrolase activities. However, products involved in transmembrane transporter activity only appeared during maturation. In the atretic ovary, the majority of products were associated with ion and lipid binding.

\section{Vitellogenesis}

In the vitellogenic ovary, 34 and 12 transcripts were found to be up- and down-regulated, respectively, relative to previtellogenic ovaries; 35 had a significant hit in Swiss-Prot database (Table 1). The most highly up-regulated transcripts corresponded to selenoprotein W2a (sepw2a), hypothetical $18 \mathrm{~K}$ protein from Carassius auratus mitochondrion, muscle-specific beta 1 integrin binding protein 2 (mibp2), zona pellucida protein 3 (zp3), cytochrome c oxidase subunit I ( $\operatorname{cox} 1)$, cytochrome b $(c y t b)$, cytosolic heat shock protein 90 beta (hsp90b), NADH dehydrogenase subunit 3 (nd3) and 1 (nd1), and beta actin 1 (bactin1). The sequence similarity of clone pgsP0015N21 to tilapia (Oreochromis mossambicus) sepw2a was low (4E-04) possibly because its nucleotide sequence only covered the $\mathrm{C}$ terminus of tilapia sepw $2 a$.

Other up-regulated genes in the vitellogenic ovary, but at lower levels, were additional components of the cytoskeleton, such as alpha actin (actc1l), keratin 8 (krt8), tropomyosin1-1 (tpm1-1), myosin (myh11), and transgelin (tagln), or of intracellular signaling pathways, such as Ras homolog member G (rhog), a novel protein similar to serum/glucocorticoid regulated kinase ( $s g k$ ) also found in zebrafish, and inositol monophosphatase 3 (impa3). Proteolytic complexes and enzymes, such as protein arginine methyltransferase (prmt1), acetyl-coenzyme A acyltransferase 2 (acaa2), and creatine kinase $(c k b)$, and the putative homeodomain transcription factor 1 (phtf1), transducer of ERBB2 (tob1a), neurexin 1a (nrxn1a) and thrombospondin $4 \mathrm{~b}(t h b s 4 b)$ were also up-regulated in vitellogenic ovaries. 
Table I: Transcripts regulated in vitellogenic ovary relative to previtellogenic ovary

\begin{tabular}{|c|c|c|c|c|c|c|c|}
\hline Clone ID & $\begin{array}{l}\text { GenBank } \\
\text { accession }\end{array}$ & $\mathbf{F C a}$ & $\begin{array}{l}\text { UniProtKB/ } \\
\text { TrEMBL entry }\end{array}$ & Swiss-Prot hit & $\begin{array}{l}\text { BLAST } \\
\text { E-value }\end{array}$ & $\begin{array}{c}\text { Length } \\
\text { (\% identity) }\end{array}$ & Gene Symbol \\
\hline PgsP00I5N2I & $\underline{F F 286629}$ & +5.13 & Q3ZLC7 & $\begin{array}{l}\text { Selenoprotein W2a } \\
\text { [Oreochromis mossambicus] }\end{array}$ & $4 \mathrm{E}-04$ & $27(70 \%)$ & sepw2a \\
\hline PgsP0003P2I & $\underline{\mathrm{FF} 282633}$ & +4.64 & $\mathrm{JCl} 348$ & $\begin{array}{l}\text { Hypothetical } 18 \mathrm{~K} \text { protein, } \\
\text { mitochondrion [Carassius } \\
\text { auratus] }\end{array}$ & $2 \mathrm{E}-06$ & $40(70 \%)$ & \\
\hline PgsP0005D08 & $\underline{F F 283023}$ & +4.49 & & Unknown & & & \\
\hline PgsP0017G22 & $\underline{F F 287169}$ & +3.94 & Q0KJI4 & $\begin{array}{l}\text { Cytochrome c oxidase } \\
\text { subunit I [Solea senegalensis] }\end{array}$ & $3 E-84$ & $230(92 \%)$ & $\operatorname{cox} I$ \\
\hline pgsP0029LI4 & $\underline{F F 291468}$ & +3.67 & Q7ZUR6 & $\begin{array}{l}\text { Muscle-specific beta I } \\
\text { integrin binding protein } 2 \\
\text { [Danio rerio] }\end{array}$ & $2 \mathrm{E}-83$ & $192(78 \%)$ & $\operatorname{mibp} 2$ \\
\hline PgsP00I8DII & $\underline{F F 287436}$ & +3.56 & Q75v54 & $\begin{array}{l}\text { Cytochrome b }[\mathrm{S} . \\
\text { senegalensis] }\end{array}$ & $8 \mathrm{E}-74$ & $165(93 \%)$ & cytb \\
\hline pgsP0019B22 & FF287743 & +3.34 & A8R7E8 & $\begin{array}{l}\text { Cytosolic heat shock } \\
\text { protein } 90 \text { beta }[S . \\
\text { senegalensis] }\end{array}$ & $6 \mathrm{E}-70$ & $133(100 \%)$ & hsp90b \\
\hline PgsP0008AII & $\underline{F F 283945}$ & +3.21 & Q0KJ09 & $\begin{array}{l}\text { NADH dehydrogenase } \\
\text { subunit } 3[\text { S. senegalensis] }\end{array}$ & IE-43 & III (84\%) & $n d 3$ \\
\hline PgsP0003A24 & $\underline{F F 282359}$ & +3.00 & BIB560 & $\begin{array}{l}\text { Beta actin isoform I [S. } \\
\text { senegalensis] }\end{array}$ & $4 \mathrm{E}-22$ & $5 \mathrm{I}(100 \%)$ & bactin I \\
\hline pgsP0020D03 & $\underline{F F 288118}$ & +2.93 & P79893 & $\begin{array}{l}\text { Chorion protein (zona } \\
\text { protein 3) [Sparus aurata] }\end{array}$ & $8 \mathrm{E}-69$ & $160(70 \%)$ & $z p 3$ \\
\hline PgsP002IP08 & FF288738 & +2.69 & Q0KJI6 & $\begin{array}{l}\text { NADH dehydrogenase } \\
\text { subunit I [S. senegalensis] }\end{array}$ & $2 \mathrm{E}-124$ & $280(88 \%)$ & ndl \\
\hline PgsP00।3B।5 & FF285673 & +2.62 & Q7SZRI & $\begin{array}{l}\text { Transducer of ERBB2, Ia } \\
\text { [Danio rerio] }\end{array}$ & IE-57 & $175(77 \%)$ & tobla \\
\hline PgsP0007G02 & $\underline{F F 283724}$ & +2.59 & Q6IQR3 & Alpha actin [D. rerio] & $7 E-123$ & $282(83 \%)$ & actcll \\
\hline PgsP0025NOI & FF290105 & +2.47 & Q805DI & $\begin{array}{l}\text { Tropomyosin I-I [Takifugu } \\
\text { rubripes] }\end{array}$ & IE-19 & $55(90 \%)$ & tpm I-I \\
\hline PgsP0029A20 & FF291229 & +2.41 & Q0P4B4 & Transgelin [D. rerio] & $4 \mathrm{E}-60$ & $|4|(79 \%)$ & tagln \\
\hline PgsP0028P05 & FF291191 & +2.29 & Q6PFN7 & $\begin{array}{l}\text { Protein arginine } \\
\text { methyltransferase I [D. } \\
\text { rerio] }\end{array}$ & IE- 167 & 308 (95\%) & prmtl \\
\hline pgsP00I5D24 & FF286403 & +2.12 & & Unknown & & & \\
\hline PgsP0020005 & FF288363 & +2.11 & & Unknown & & & \\
\hline PgsP00I4A08 & $\underline{F F 285984}$ & +2.09 & & Unknown & & & \\
\hline PgsP0029Cl6 & FF29127I & +1.99 & AIXQXO & Neurexin Ia [D. rerio] & $3 \mathrm{E}-132$ & $266(84 \%)$ & $n r x n l a$ \\
\hline PgsP0OI5HI5 & $\underline{F F 286488}$ & +1.98 & & Unknown & & & \\
\hline PgsP0030124 & FF291759 & +1.96 & & Unknown & & & \\
\hline PgsP0029LI9 & FF29|472 & +1.92 & Q8JGW0 & $\begin{array}{l}\text { Thrombospondin } 4 \mathrm{~b}[D . \\
\text { rerio] }\end{array}$ & IE- 100 & 175 (97\%) & thbs $4 b$ \\
\hline PgsP0004D0I & FF282692 & +1.93 & Q5ZMG8 & $\begin{array}{l}\text { Ras homolog member G } \\
\text { (rho G) [Gallus gallus] }\end{array}$ & $2 \mathrm{E}-90$ & $162(93 \%)$ & rhog \\
\hline PgsP00I7K07 & $\underline{F F 287242}$ & +1.90 & Q6NWF6 & $\begin{array}{l}\text { Keratin, type II cytoskeletal } \\
8 \text { [D. rerio] }\end{array}$ & $4 \mathrm{E}-33$ & $79(94 \%)$ & krt8 \\
\hline PgsP0028FI 4 & FF290985 & +1.89 & Q9UMS5 & $\begin{array}{l}\text { Putative homeodomain } \\
\text { transcription factor I [Homo } \\
\text { sapiens] }\end{array}$ & $4 \mathrm{E}-56$ & I44 (70\%) & phtfl \\
\hline PgsP0016G07 & FF286813 & +1.88 & & Unknown & & & \\
\hline PgsP0009A I7 & FF284280 & +1.87 & Q2YDR3 & $\begin{array}{l}\text { Inositol monophosphatase } 3 \\
\text { [D. rerio] }\end{array}$ & $6 \mathrm{E}-13$ & 74 (84\%) & impa3 \\
\hline PgsP0027DI4 & FF290593 & +1.85 & Q8AY63 & $\begin{array}{l}\text { Creatine kinase, brain }[D . \\
\text { rerio] }\end{array}$ & $7 E-84$ & $179(90 \%)$ & $c k b$ \\
\hline PgsP0020F22 & FF288I78 & +1.82 & Q4U0S2 & $\begin{array}{l}\text { Smooth muscle myosin } \\
\text { heavy chain }[D \text {. rerio }]\end{array}$ & IE-97 & $180(87 \%)$ & myh II \\
\hline pgsP0027C08 & $\underline{F F 290565}$ & +1.80 & Q3T0R7 & $\begin{array}{l}\text { 3-Ketoacyl-CoA thiolase, } \\
\text { mitochondrial [Bos taurus] }\end{array}$ & IE-30 & 76 (82\%) & acaa2 \\
\hline pgsP0028D08 & $\underline{F F 290934}$ & +1.76 & & Unknown & & & \\
\hline PgsP0008G08 & $\underline{F F 284066}$ & +1.74 & & Unknown & & & \\
\hline
\end{tabular}


Table I: Transcripts regulated in vitellogenic ovary relative to previtellogenic ovary (Continued)

\begin{tabular}{|c|c|c|c|c|c|c|c|}
\hline PgsP000IG2I & $\underline{F F 281897}$ & +1.74 & A3KQ53 & $\begin{array}{l}\text { Novel protein similar to } \\
\text { vertebrate serum/ } \\
\text { glucocorticoid regulated } \\
\text { kinase (SGK) [D. rerio] }\end{array}$ & $2 \mathrm{E}-45$ & $90(81 \%)$ & $s g k$ \\
\hline PgsP00I3L08 & $\underline{F F 285880}$ & -5.32 & QIL8U4 & $\begin{array}{l}\text { Novel protein similar to } \\
\text { vertebrate CDC-like kinase } \\
2(C L K 2)[D \text {. rerio] }\end{array}$ & $4 \mathrm{E}-91$ & $184(87 \%)$ & si:ch2 I I-8 Ia5.7 \\
\hline PgsP0028DI8 & $\underline{F F 290943}$ & -4.80 & Q6PHK4 & $\begin{array}{l}\text { Alanine-glyoxylate } \\
\text { aminotransferase [D. rerio] }\end{array}$ & $2 \mathrm{E}-64$ & $150(76 \%)$ & agxt \\
\hline PgsP0020C06 & FF288101 & -3.58 & & Unknown & & & \\
\hline $\mathrm{PgsP} 0028 \mathrm{HII}$ & FF291026 & -3.17 & Q7T376 & $\begin{array}{l}\text { Novel protein similar to } \\
\text { vertebrate CD } 53 \text { molecule } \\
\text { [D. rerio] }\end{array}$ & $8 \mathrm{E}-24$ & $92(55 \%)$ & zgc:6405I \\
\hline PgsP0027FI8 & FF290638 & -2.23 & & Unknown & & & \\
\hline PgsP0028J05 & FF291062 & -2.22 & Q8HXG6 & $\begin{array}{l}\text { NADH dehydrogenase } \\
\text { [ubiquinone] I alpha } \\
\text { subcomplex subunit II [B. } \\
\text { taurus] }\end{array}$ & $2 \mathrm{E}-23$ & $133(44 \%)$ & ndufal I \\
\hline PgsP0008II2 & FF284II4 & -2.12 & Q6NY77 & $\begin{array}{l}\text { Dihydrodipicolinate } \\
\text { synthase-like, mitochondrial } \\
\text { [D. rerio] }\end{array}$ & $2 E-66$ & $172(77 \%)$ & zgc:77082 \\
\hline PgsP0007P0I & FF283914 & -2.12 & Q7TQ08 & Scinderin [Rattus norvegicus] & $3 \mathrm{E}-79$ & $193(70 \%)$ & scin \\
\hline PgsP0009H07 & FF284426 & -2.10 & Q2TATI & $\begin{array}{l}\text { Hypothetical protein } \\
\text { LOC735233 [Xenopus laevis] }\end{array}$ & IE-24 & $166(43 \%)$ & MGCI30935 \\
\hline PgsP0016EI5 & FF286777 & -2.06 & Q8UW64 & $\begin{array}{l}\text { Proteasome subunit beta } \\
\text { type-9 [Oryzias latipes] }\end{array}$ & $3 \mathrm{E}-4 \mathrm{I}$ & 98 (84\%) & psmb9a \\
\hline PgsP0004Al4 & FF282647 & -2.01 & Q5JBII & $\begin{array}{l}\text { Carboxypeptidase } \mathrm{H} \\
\text { [Paralichthys olivaceus] }\end{array}$ & $4 \mathrm{E}-58$ & $133(94 \%)$ & $c p h$ \\
\hline PgsP00I $2 M I 2$ & FF285564 & -1.89 & Q6ZM96 & $\begin{array}{l}\text { Coiled-coil domain } \\
\text { containing 90B [D. rerio] }\end{array}$ & $\mathrm{IE}-22$ & 107 (61\%) & $c c d c 90 b$ \\
\hline
\end{tabular}

aFold change; b Extent of BLASTX hit aligned region (in amino acids), and percent identity over the aligned region.

Two transcripts similar to CDC-like kinase 2 (si:ch21181a5.7) and CD53 cell surface glycoprotein (zgc:64051), as well as alanine-glyoxylate aminotransferase (agxt), were the most down-regulated genes during vitellogenesis. Other down-regulated genes included the actin-binding protein scinderin (scin), mitochondrial enzymes, such as NADH dehydrogenase (ubiquinone) 1 alpha subcomplex subunit 11 (ndufa11) and a dihydrodipicolinate synthaselike enzyme, proteolytic complexes and enzymes, as proteasome subunit beta type-9 precursor (psmb9a) and carboxypeptidase $\mathrm{H}(c p h)$, a hypothetical protein-encoding gene also found in Xenopus laevis (LOC735233), and a coiled-coil domain containing 90B novel product (ccdc90b).

\section{Ovarian maturation}

Microarray analysis detected 26 up-regulated and 20 down-regulated transcripts in maturing/mature ovaries relative to vitellogenic ovaries, and 32 transcripts could be annotated (Table 2). The most highly up-regulated transcript corresponded to an EST showing sequence similarity to the amphioxus (Branchiostoma floridae) BRAFLDRAFT_128798 gene, which encodes an hypothetical protein with inferred cysteinyl-tRNA aminoacylation activity. However, the BLAST E-value for the similarity of Senegalese sole clone pgsP0012B12 to this protein was relatively low (2E-14), and therefore conclusive annota- tion will require the cloning of the sole full-length cDNA. Other highly up-regulated transcripts encoded $\mathrm{Na}^{+} / \mathrm{K}^{+}$ ATPase subunits, such as the beta subunit 1a (atp1b1a), alpha subunit 1 (atp1a1) and another isoform of the beta subunit $(a t p b)$, and alpha-2-macroglobulin $(a 2 m)$.

Cytoskeletal proteins, myosin 10 (myo10) and type II keratin E3-like protein, and proteins involved in transcriptional and translational responses, such as makorin RING zinc finger protein 1a ( $m k r n 1)$ and ribosomal protein L36 (rpl36), were other up-regulated transcripts in mature ovaries. Interestingly, a regulator of vesicular traffic, novel protein similar to vertebrate ADP-ribosylation factor 4 and extended synaptotagmin-2-A (e-syt2-a), was also upregulated. Other transcripts were retinoic acid receptor responder protein 3 (rarres3), thioredoxin interacting protein (txnip), mitogen-activated protein kinase p38delta (mapk13), cytochrome c oxidase subunit I (cox1), UDPglucose dehydrogenase (ugdh), and myeloid-associated differentiation marker homolog (myadm). Some transcripts that were up-regulated during vitellogenesis showed a further increase during maturation, such as tob1 $a$ and $n d 1$.

During ovarian maturation, more genes appeared to be down-regulated than during vitellogenesis. Among those transcripts, we found type II Na/Pi cotransport system 
Table 2: Transcripts regulated in mature ovary relative to vitellogenic ovary

\begin{tabular}{|c|c|c|c|c|c|c|c|}
\hline Clone ID & $\begin{array}{l}\text { GenBank } \\
\text { accession }\end{array}$ & FCa & $\begin{array}{l}\text { UniProtKB/ } \\
\text { TrEMBL entry }\end{array}$ & Swiss-Prot hit & $\begin{array}{l}\text { BLAST } \\
\text { E-value }^{\text {b }}\end{array}$ & $\begin{array}{c}\text { Length } \\
\text { (\% identity) }\end{array}$ & Gene Symbol \\
\hline PgsP00 I 2B I 2 & $\underline{F F 285326}$ & +4.54 & B6NTA9 & $\begin{array}{l}\text { Hypothetical protein } \\
\text { BRAFLDRAFT_I } 28798 \\
\text { [Branchiostoma floridae] }\end{array}$ & $2 \mathrm{E}-14$ & $107(4 \mid \%)$ & $\begin{array}{c}\text { BRAFLDRAFT_I } 28 \\
798\end{array}$ \\
\hline PgsP00I6M08 & $\underline{F F 286940}$ & +2.56 & Q6NYH5 & $\begin{array}{l}\text { ATPase, } \mathrm{Na}+\mathrm{V} / \mathrm{K}+ \\
\text { transporting, beta la } \\
\text { polypeptide [Danio rerio] }\end{array}$ & $2 \mathrm{E}-10$ & 39 (76\%) & $a t p / b / a$ \\
\hline PgsP00I3HI6 & $\underline{F F 285803}$ & +2.51 & Q8AY58 & $\begin{array}{l}\text { Sodium/potassium ATPase } \\
\text { alpha subunit isoform I } \\
\text { [Fundulus heteroclitus] }\end{array}$ & $2 \mathrm{E}-118$ & $213(95 \%)$ & atplal \\
\hline PgsP00I7BI5 & $\underline{F F 287055}$ & +2.22 & Q67EX5 & $\begin{array}{l}\text { Alpha-2-macroglobulin } \\
\text { [Sparus aurata] }\end{array}$ & $4 \mathrm{E}-8 \mathrm{I}$ & $221(70 \%)$ & $a 2 m$ \\
\hline PgsP0023110 & FF289288 & +1.97 & Q56V59 & $\begin{array}{l}\text { Sodium potassium ATPase } \\
\text { beta subunit [Rhabdosargus } \\
\text { sarba] }\end{array}$ & IE-79 & $216(62 \%)$ & $a t p b$ \\
\hline PgsP0030LI6 & FF291822 & +1.86 & Q694W8 & Myosin I0 [Xenopus laevis] & $5 E-32$ & $78(83 \%)$ & myolo \\
\hline PgsP0027A07 & FF290524 & +1.75 & Q9UL19 & $\begin{array}{l}\text { Retinoic acid receptor } \\
\text { responder protein } 3 \text { [Homo } \\
\text { sapiens] }\end{array}$ & $5 \mathrm{E}-15$ & $108(40 \%)$ & rarres3 \\
\hline PgsP0007CII & $\underline{F F 283652}$ & +1.69 & & Unknown & & & \\
\hline PgsP0009C03 & $\overline{\mathrm{FF} 284311}$ & +1.64 & & Unknown & & & \\
\hline PgsP0022M06 & $\underline{F F 289024}$ & +1.64 & Q7ZWB6 & $\begin{array}{l}\text { Thioredoxin interacting } \\
\text { protein [D. rerio] }\end{array}$ & $2 \mathrm{E}-108$ & $296(68 \%)$ & txnip \\
\hline PgsP00।3B।5 & FF285673 & +1.61 & Q7SZRI & $\begin{array}{l}\text { Transducer of ERBB2, la } \\
\text { [D. rerio] }\end{array}$ & IE-57 & $175(77 \%)$ & tobla \\
\hline PgsP0022B09 & FF288784 & +1.60 & A9ZTB5 & $\begin{array}{l}\text { Ribosomal protein L36 } \\
\text { [Solea senegalensis] }\end{array}$ & $3 E-46$ & $94(100 \%)$ & $r p / 36$ \\
\hline PgsP0003BI4 & $\underline{F F 282371}$ & +1.57 & & Unknown & & & \\
\hline PgsP0027GII & $\overline{\mathrm{FF} 290655}$ & +1.55 & & Unknown & & & \\
\hline PgsP0019DI3 & FF287779 & +1.45 & & Unknown & & & \\
\hline PgsP0005HI 4 & FF283112 & +1.42 & & Unknown & & & \\
\hline PgsP000INI7 & $\overline{\mathrm{FF} 282035}$ & +1.41 & Q5FWL4 & $\begin{array}{l}\text { Extended synaptotagmin-2- } \\
\mathrm{A}[\mathrm{X} \text {. laevis }]\end{array}$ & $7 E-58$ & 144 (74\%) & e-syt2-a \\
\hline PgsP0022B24 & FF288799 & +1.40 & Q5NUI4 & $\begin{array}{l}\text { Makorin RING zinc finger } \\
\text { protein Ia [Takifugu } \\
\text { rubripes] }\end{array}$ & $4 \mathrm{E}-64$ & $224(50 \%)$ & $m k r n l$ \\
\hline PgsP0022J20 & $\underline{F F 288970}$ & +1.40 & Q9WTY9 & $\begin{array}{l}\text { Mitogen-activated protein } \\
\text { kinase } \mathrm{p} 38 \text { delta [Rattus } \\
\text { norvegicus] }\end{array}$ & $3 \mathrm{E}-29$ & 127 (49\%) & mapk /3 \\
\hline PgsP0027B20 & FF290555 & +1.39 & Q6P5M5 & $\begin{array}{l}\text { Novel protein similar to } \\
\text { vertebrate ADP- } \\
\text { ribosylation factor } 4[D . \\
\text { rerio] }\end{array}$ & $6 \mathrm{E}-76$ & 174 (86\%) & zgc:77650 \\
\hline PgsP002IP08 & $\underline{F F 288738}$ & +1.39 & Q0KJI6 & $\begin{array}{l}\mathrm{NADH} \text { dehydrogenase } \\
\text { subunit I [S. senegalensis] }\end{array}$ & $2 \mathrm{E}-124$ & $280(88 \%)$ & ndl \\
\hline PgsP00I7G22 & FF287169 & +1.38 & Q0KJI4 & $\begin{array}{l}\text { Cytochrome c oxidase } \\
\text { subunit I [S. senegalensis] }\end{array}$ & $3 E-84$ & $230(92 \%)$ & $\operatorname{cox} I$ \\
\hline pgsP0007O02 & FF283892 & +1.37 & A8WGP7 & $\begin{array}{l}\text { UDP-glucose } \\
\text { dehydrogenase [D. rerio] }\end{array}$ & IE-38 & $104(75 \%)$ & ugdh \\
\hline PgsP00I8PI5 & FF287693 & +1.36 & Q63ZU3 & $\begin{array}{l}\text { Myeloid-associated } \\
\text { differentiation marker } \\
\text { homolog [X. laevis] }\end{array}$ & $3 \mathrm{E}-15$ & $93(47 \%)$ & myadm \\
\hline PgsP0029BI2 & FF291245 & +1.36 & Q4QY72 & $\begin{array}{l}\text { Type II keratin E3-like } \\
\text { protein }[\text { S. aurata] }\end{array}$ & $4 \mathrm{E}-108$ & $217(92 \%)$ & \\
\hline PgsP0006O02 & FF283575 & +1.32 & & Unknown & & & \\
\hline pgsP0027D07 & $\overline{\mathrm{FF} 290586}$ & -2.49 & & Unknown & & & \\
\hline PgsP00I7L02 & $\overline{F F 287257}$ & -2.31 & Q9PTQ8 & $\begin{array}{l}\text { Type II Na/Pi cotransport } \\
\text { system protein [D. rerio] }\end{array}$ & $7 E-32$ & $128(73 \%)$ & slc34a2a \\
\hline PgsP0023FI3 & FF289226 & -2.11 & Q5M90I & $\begin{array}{l}\text { Myosin binding protein } \mathrm{H} \\
\text { [X. tropicalis] }\end{array}$ & IE-46 & $139(64 \%)$ & mybph \\
\hline PgsP0002P23 & FF282343 & -1.80 & & Unknown & & & \\
\hline PgsP00I9F07 & $\overline{\mathrm{FF} 287815}$ & -1.72 & Q5XWB3 & Ubiquitous gelsolin [D. rerio] & $2 \mathrm{E}-8 \mathrm{I}$ & $192(72 \%)$ & gsna \\
\hline
\end{tabular}


Table 2: Transcripts regulated in mature ovary relative to vitellogenic ovary (Continued)

\begin{tabular}{|c|c|c|c|c|c|c|c|}
\hline PgsP0009JI5 & $\underline{F F 284472}$ & -1.71 & Q007T0 & $\begin{array}{l}\text { Succinate dehydrogenase } \\
\text { [ubiquinone] iron-sulfur } \\
\text { subunit, mitochondrial [Sus } \\
\text { scrofa] }\end{array}$ & IE-08 & 30 (93\%) & $s d h b$ \\
\hline PgsP0023HI2 & FF289266 & -1.71 & A2CEC 9 & $\begin{array}{l}\text { Novel protein similar to } \\
\text { centaurin, delta } 2[D \text {. rerio] }\end{array}$ & $5 \mathrm{E}-36$ & $272(36 \%)$ & LOCI00005008 \\
\hline PgsP0009A I7 & FF284280 & -1.70 & Q28CL4 & $\begin{array}{l}\text { Inositol monophosphatase } 3 \\
\text { [X. tropicalis] }\end{array}$ & $7 E-12$ & 66 (7I\%) & impa3 \\
\hline pgsP0024D08 & FF289528 & -1.59 & & Unknown & & & \\
\hline PgsP0022O08 & FF289072 & -1.53 & Q7SXH8 & $\begin{array}{l}\text { Coagulation factor II } \\
\text { (thrombin) [D. rerio] }\end{array}$ & $4 \mathrm{E}-88$ & 140 (76\%) & $f 2$ \\
\hline PgsP0010AI5 & FF284617 & -1.48 & Q6J514 & $\begin{array}{l}\text { Monocytic leukemia zinc } \\
\text { finger protein [D. rerio] }\end{array}$ & $3 \mathrm{E}-22$ & $96(90 \%)$ & myst3 \\
\hline PgsP0029E I 6 & FF291319 & -1.46 & Q6P8F7 & $\begin{array}{l}\text { Fructose-I,6- } \\
\text { bisphosphatase }[X . \\
\text { tropicalis }]\end{array}$ & $\mid E-132$ & $23 \mid(77 \%)$ & $f b p l$ \\
\hline pgsP00।9H08 & FF287862 & -1.43 & XP_001920639 & $\begin{array}{l}\text { Similar to apolipoprotein } L, \\
3[\text { [D. rerio] }\end{array}$ & $9 \mathrm{E}-60$ & II 5 (72\%) & $10 C 100150119$ \\
\hline PgsP00I5B20 & $\underline{F F 286358}$ & -1.43 & Q8AYLI & $\begin{array}{l}\text { Zona pellucida C2 [Oryzias } \\
\text { latipes] }\end{array}$ & $9 \mathrm{E}-69$ & $161(67 \%)$ & $z p c 2$ \\
\hline PgsP00। $8 J 23$ & FF287569 & -1.40 & B2R4S9 & $\begin{array}{l}\text { Histone I, H2bc, isoform } \\
\text { CRA_a [H. sapiens] }\end{array}$ & $3 E-35$ & 77 (97\%) & hist / h2be \\
\hline PgsP0029D07 & $\underline{F F 291286}$ & -1.39 & P62260 & $\begin{array}{l}\text { 14-3-3 protein epsilon }[R . \\
\text { norvegicus] }\end{array}$ & $2 \mathrm{E}-20$ & $65(76 \%)$ & ywhae \\
\hline PgsP0030124 & FF291759 & -1.39 & & Unknown & & & \\
\hline PgsP0013O20 & FF285955 & -1.38 & & Unknown & & & \\
\hline PgsP0020JII & FF288260 & -1.36 & & Unknown & & & \\
\hline PgsP0023LI 5 & FF289356 & -1.33 & & Unknown & & & \\
\hline
\end{tabular}

aFold change; ${ }^{b}$ Extent of BLASTX hit aligned region (in amino acids), and percent identity over the aligned region.

protein $($ slc34a2a) and enzymes such as succinate dehydrogenase complex subunit B $(s d h b)$ and fructose-1,6bisphosphatase ( $f b p 1)$, involved in carbohydrate metabolism, and impa3, which was up-regulated during vitellogenesis. Transcript abundance was also reduced for some components and regulators of the cytoskeleton, such as myosin binding protein $\mathrm{H}(m y b p h)$, gelsolin (gsna), and centaurin delta 2-like (LOC100005008). Other transcripts were thrombin $(f 2)$, monocytic leukemia zinc finger protein (myst3), 14-3-3 protein epsilon (ywhae), an apolipoprotein L-like protein (LOC100150119), zona pellucida C2 (zpc2) and histone H2B (hist1h2be). These mRNAs are potentially implicated in proteolysis $(f 2)$, transcription and signal transduction regulation ( $m y s t 3$ and ywhae, respectively), lipid transport (LOC100150119), formation of the zona radiata $(z p c 2)$, and chromatin compaction (hist1h2be).

\section{Follicular atresia}

The comparison of ovaries undergoing follicular atresia $v s$. vitellogenic and mature ovaries revealed the up- and down-regulation of 10 and 16 transcripts, respectively, and 18 transcripts could be annotated (Table 3). One of these transcripts (GenBank accession number FF286365), which was the most highly up-regulated, had sequence similarity to two unknown predicted proteins from gilthead sea bream (Sparus aurata) and the puffer fish Tetraodon nigroviridis (BLAST E-values of 9E-08 and 3E-04, respectively). This EST apparently encoded a full-length polypeptide which shared $25 \%$ identity with a protein named gastrula-specific embryonic protein 1 found in the orange-spotted grouper (Epinephelus coioides). The corresponding cDNA clone (pgsP0015C05) was then sequenced in full-length, and the presence of conserved motifs in its deduced amino acid sequence was investigated. These analyses, together with a preliminary phylogenetic reconstruction, clearly indicated that sole FF286365 encoded an ortholog of apolipoprotein C-I (apoc1) (Additional file 2). The nucleotide and amino acid sequence of this cDNA was deposited in GenBank with accession number EU835856.

Other transcripts also significantly up-regulated in atretic ovaries were leukocyte cell-derived chemotaxin 2 (lect), thrombospondin (thbs), heme-binding protein 2 (hebp2), apolipoprotein A-I (apoa1), S100-like calcium binding protein (s100), and enolase (eno3). The S100-encoding EST (pgsP0020M08) was a full-length cDNA which allowed further analysis of its deduced amino acid sequence. The analysis indicated that this transcript belongs to the S100a10 subgroup of the EF-Hand calcium-binding proteins superfamily (Additional file 3).

Regarding down-regulated transcripts, BRAFLDRAFT_128798 and $a 2 m$ showed the strongest repression in atretic ovaries, which interestingly were 
Table 3: Transcripts regulated in atretic ovary relative to vitellogenic/mature ovary

\begin{tabular}{|c|c|c|c|c|c|c|c|}
\hline Clone ID & $\begin{array}{l}\text { GenBank } \\
\text { accession }\end{array}$ & $\mathbf{F C a}$ & $\begin{array}{l}\text { UniProtKB/ } \\
\text { TrEMBL entry }\end{array}$ & Swiss-Prot hit & $\begin{array}{l}\text { BLAST } \\
\text { E-value }\end{array}$ & $\begin{array}{c}\text { Length } \\
\text { (\% identity) }\end{array}$ & Gene Symbol \\
\hline PgsP0015C05 & $\underline{F F 286365}$ & +5.13 & B6DUH2 & $\begin{array}{l}\text { Apolipoprotein C-I } \\
\text { [Hemibarbus mylodon] }\end{array}$ & IE-08 & $64(46 \%)$ & apocl \\
\hline PgsP0002L08 & FF282262 & +3.36 & & Unknown & & & \\
\hline pgsP0007K07 & FF283810 & +2.90 & A8D3J2 & $\begin{array}{l}\text { Leukocyte cell-derived } \\
\text { chemotaxin } 2 \text { [Lates } \\
\text { calcarifer] }\end{array}$ & $3 E-53$ & $129(77 \%)$ & lect2 \\
\hline $\mathrm{PgsP0010012}$ & $\underline{F F 284909}$ & +2.35 & Q28I78 & $\begin{array}{l}\text { Thrombospondin [Bos } \\
\text { taurus] }\end{array}$ & IE-27 & $76(72 \%)$ & thbs \\
\hline PgsP0022K2I & $\underline{F F 288994}$ & +2.05 & B5X719 & $\begin{array}{l}\text { Heme-binding protein } 2 \\
\text { [Salmo salar] }\end{array}$ & $2 \mathrm{E}-32$ & $85(40 \%)$ & hebp2 \\
\hline PgsP0008CI7 & FF283994 & +1.93 & Q5KSU5 & $\begin{array}{l}\text { Apolipoprotein A-I } \\
\text { [Takifugu rubripes] }\end{array}$ & $7 E-53$ & 197 (49\%) & apoal \\
\hline PgsP0020M08 & FF288324 & +1.84 & A8HG28 & $\begin{array}{l}\text { SI00-like calcium binding } \\
\text { protein [Epinephelus } \\
\text { coioides] }\end{array}$ & $6 \mathrm{E}-38$ & $101(74 \%)$ & s100 \\
\hline PgsP0005K I6 & FF283180 & +1.62 & Q6THI4 & Enolase [Danio rerio] & $|E-9|$ & 190 (92\%) & eno3 \\
\hline PgsP0019FI7 & $\underline{F F 287823}$ & +1.62 & & Unknown & & & \\
\hline PgsP00I 3020 & $\overline{\mathrm{FF} 285955}$ & +1.49 & & Unknown & & & \\
\hline $\mathrm{PgsP} 0012 \mathrm{~B} / 2$ & FF285326 & -4.01 & B6NTA9 & $\begin{array}{l}\text { Hypothetical protein } \\
\text { BRAFLDRAFT_I28798 } \\
\text { [Branchiostoma floridae] }\end{array}$ & $2 \mathrm{E}-\mathrm{I} 4$ & 107 (4I\%) & $\begin{array}{c}\text { BRAFLDRAFT_I } 28 \\
798\end{array}$ \\
\hline PgsP0017BI5 & $\underline{F F 287055}$ & -2.35 & Q67EX5 & $\begin{array}{l}\text { Alpha-2-macroglobulin } \\
\text { [Sparus aurata] }\end{array}$ & $4 \mathrm{E}-8 \mathrm{I}$ & $221(70 \%)$ & $a 2 m$ \\
\hline PgsP0025P2I & $\underline{F F 290169}$ & -2.08 & XP_001519899 & $\begin{array}{l}\text { Similar to hCG } 2006161 \text {, } \\
\text { partial [Ornithorhynchus } \\
\text { anatinus] }\end{array}$ & $4 \mathrm{E}-04$ & $54(51 \%)$ & LOCI0009088I \\
\hline PgsP0006EIO & $\underline{F F 283378}$ & -1.93 & Q66KL2 & $\begin{array}{l}\text { C-terminal binding protein } \\
\text { I [Xenopus tropicalis] }\end{array}$ & $|E-6|$ & $178(78 \%)$ & $c t b p l$ \\
\hline PgsP00I3109 & FF285819 & -1.88 & & Unknown & & & \\
\hline PgsP002ILI 2 & $\underline{F F 28865 I}$ & -1.83 & Q4H447 & $\begin{array}{l}\text { Elongation factor I alpha } \\
\text { [Hyla japonica] }\end{array}$ & 0.64 & $16(69 \%)$ & efla \\
\hline pgsP0002C23 & $\underline{F F 282126}$ & -1.74 & Q90YM8 & $\begin{array}{l}\text { Cytoplasmic FMRI } \\
\text { interacting protein I } \\
\text { homolog [D. rerio] }\end{array}$ & $9 \mathrm{E}-119$ & $227(91 \%)$ & cyfipl \\
\hline PgsP00I3HI8 & FF285805 & -1.68 & XP_001340467.2 & wu:fb2If05 [D. rerio] & $2 \mathrm{E}-23$ & $112(50 \%)$ & wu:fb2 I f05 \\
\hline PgsP0024CII & $\underline{F F 289508}$ & -1.68 & & Unknown & & & \\
\hline PgsP0007E08 & FF283689 & -1.58 & B0UY57 & $\begin{array}{l}\text { Novel protein similar to } \\
\text { vertebrate RAPI } \\
\text { interacting factor homolog } \\
{[D \text {. rerio] }}\end{array}$ & $7 E-38$ & $112(76 \%)$ & rifl \\
\hline PgsP0009C03 & $\underline{F F 284311}$ & -1.58 & & Unknown & & & \\
\hline PgsP00I3B03 & $\underline{F F 285662}$ & -1.58 & & Unknown & & & \\
\hline PgsP0014AI7 & FF285991 & -1.56 & B5X408 & $\begin{array}{l}\text { Elongation of very long } \\
\text { chain fatty acids protein I } \\
\text { [S. salar] }\end{array}$ & IE-24 & $60(60 \%)$ & elovllb \\
\hline PgsP0002P23 & FF282343 & -1.56 & B5X202 & $\begin{array}{l}\text { Zinc finger protein } 576 \text { [S. } \\
\text { salar] }\end{array}$ & IE-04 & $65(41 \%)$ & znf5 76 \\
\hline pgsP0006DI8 & $\underline{F F 283364}$ & -1.55 & B5X308 & $\begin{array}{l}\text { Golgi membrane protein I } \\
\text { [S. salar] }\end{array}$ & $2 \mathrm{E}-19$ & $55(60 \%)$ & golm I \\
\hline PgsP0030GII & FF291702 & -1.55 & & Unknown & & & \\
\hline
\end{tabular}

aFold change; b Extent of BLASTX hit aligned region (in amino acids), and percent identity over the aligned region.

highly up-regulated in mature ovaries. Other reduced transcripts were potentially involved in the organization of Golgi complex, such as C-terminal binding protein 1 (ctbp1) and Golgi membrane protein 1 (golm1), or the telomeric region, such as a novel protein similar to vertebrate RAP1 interacting factor homolog (rif1), as well as in transcription and translation regulation, such as elongation factor 1 alpha (ef1a) and zinc finger protein 576 ( $z$ n576). The identity of sole FF288651 and FF282343 as ef1 $a$ and $z n f 576$, respectively, was however not conclusive since the BLAST E-values were low. Cytoplasmic FMR1 interacting protein 1 homolog (cyfip1) and elongation of 


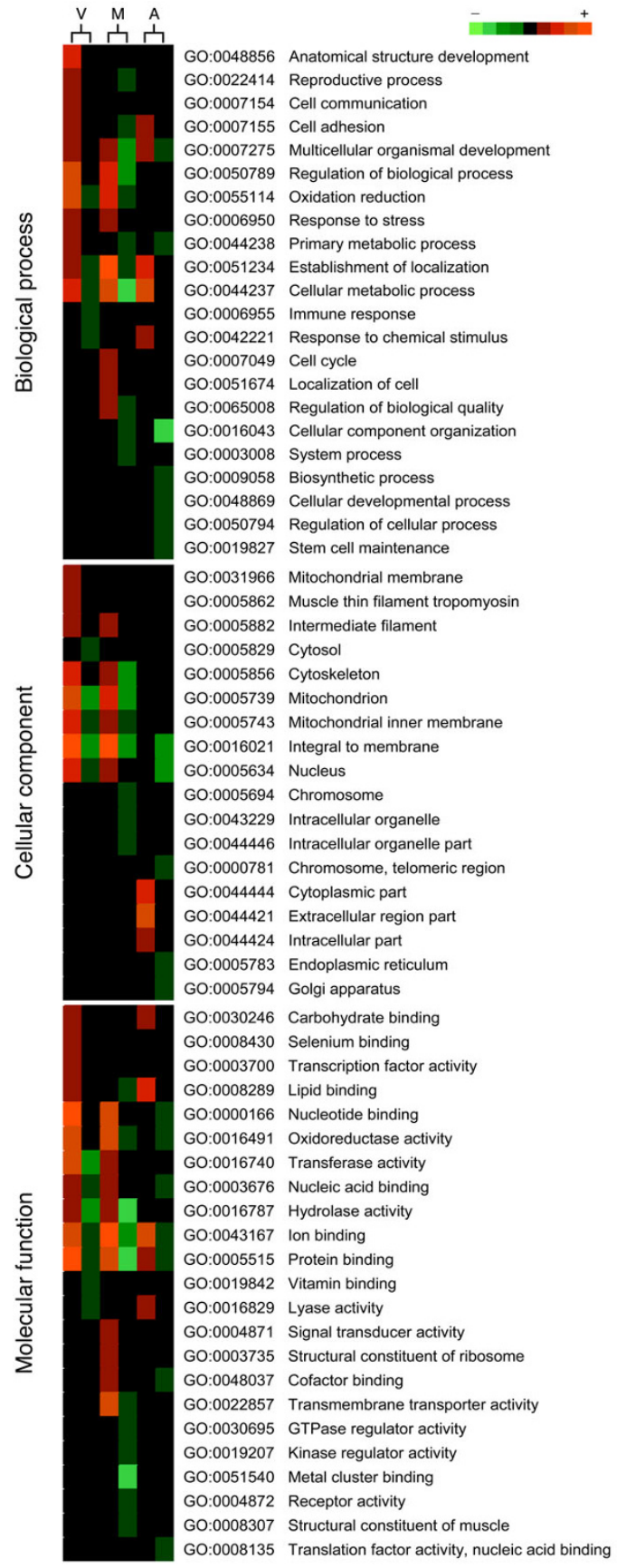

Figure 3

Gene ontology (GO) analysis of differentially expressed genes in the Senegalese sole ovary. Genes regulated during vitellogenesis $(V)$, maturation $(M)$ and atresia $(A)$ were classified according to $G O$ terms biological process (level 3), cellular component (level 5) and molecular function (level 3). For each GO term, the number of differentially expressed transcripts detected in the microarray is indicated using a color intensity scale. Red and green are used for over and under abundance, respectively, whereas black indicates no change. very long chain fatty acids protein 1 (elovl1b), which may be involved in the control of cell projections and fatty acid biosynthesis, respectively, were also down-regulated.

\section{Validation of microarray data by real-time qPCR}

A number of differentially expressed ESTs $(n=20)$ in vitellogenic, mature and atretic ovaries were further selected to verify the changes in expression by real-time quantitative RT-PCR (qPCR). The expression of all twenty genes followed the same pattern whether evaluated by microarray or qPCR (Figure 4). Two genes, tob1 $a$ and LOC100090881, were however an exception. For tob1a, a significant increase during maturation observed with the microarray could not be detected ( $p=0.78$ ) by qPCR (Figure 4B and $4 \mathrm{C})$, whereas the significant down-regulation of LOC100090881 during atresia could not be confirmed ( $p$ $=0.88$ ) by qPCR (Figure 4C). All other genes showed in general a similar relative expression pattern by both microarray and qPCR, resulting in an overall success rate of $91 \%$ ( 2 inconsistencies out of 22 comparisons, since tob1a and BRAFLDRAFT_128798 were significantly regulated both during vitellogenesis and atresia by microarray analysis). For $a 2 m$, however, the FC determined with the array (2.35) was about 10 times lower than that measured by qPCR (18.38), which is a known phenomenon observed in oligo-arrays when background subtraction is not performed (as in the present study) [32]. Usually, a two-fold change is considered as the cut-off around which microarray and qRT-PCR data begin to loose correlation [33]. Finally, few transcripts that did not show significant differences in expression levels with the microarray were also selected for qPCR. These analyses did not show significant changes in the expression level consistent with the array data (data not shown).

\section{Differential expression during follicular atresia}

Some regulated transcripts in atretic ovaries relative to mature/vitellogenic ovaries, such as apoc1, apoa1, thbs, lect2, s100a10, a2m and BRAFLDRAFT_128798, were further analyzed by qPCR to investigate how broadly they might be expressed during ovarian development (Figure 5). For apoc1, thbs, s100a10, a2m and BRAFLDRAFT_128798, these analyses were also carried out on manually isolated ovarian follicles at the stages of vitellogenesis, maturation and atresia. The results confirmed that apoc1, apoa1, thbs, lect2 and s1001a10 transcripts were significantly $(p<0.05)$ up-regulated in atretic ovaries, whereas $a 2 m$ and BRAFLDRAFT_128798 transcripts were accumulated in mature ovaries and strongly down-regulated in atretic ovaries, thus demonstrating the same expression pattern as that observed with the microarray. The data also revealed that apoa1, thbs and lect 2 showed relatively high relative expression levels in previtellogenic ovaries in addition to during atresia. 
A

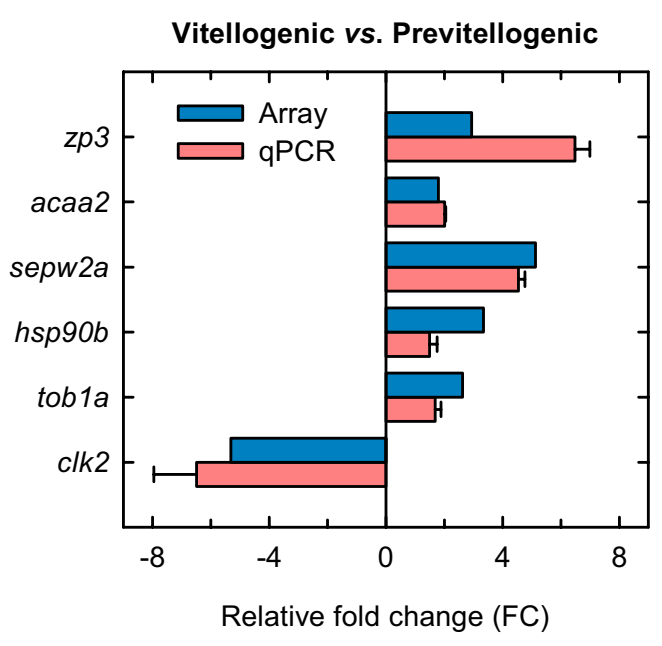

B

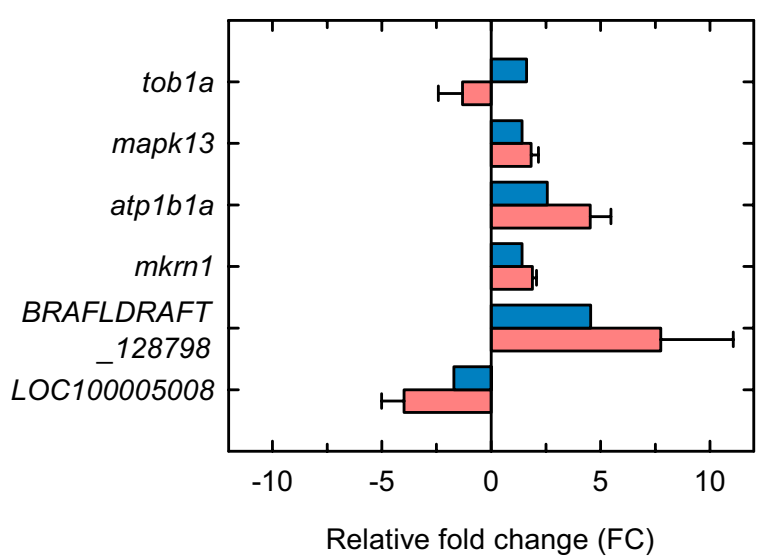

C

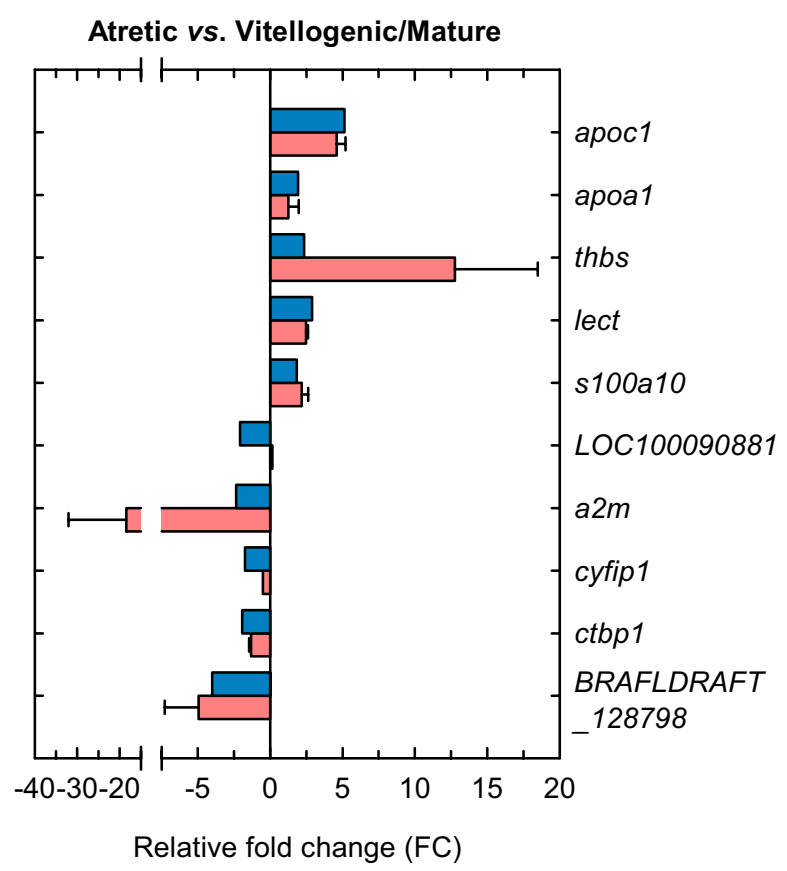

Figure 4

Real-time qPCR validation of differential expression in vitellogenic vs. previtellogenic ovaries (A), mature vs. vitellogenic ovaries (B), and atretic vs. vitellogenic/mature ovaries (C). Bar graphs represent relative fold change (FC) of selected transcripts obtained with the microarray (black bars) or by QPCR (with bars). The mean FC \pm SEM are shown for each gene. The qPCR and microarray experiments were both conducted on RNA extracted from the same three female individuals for each developmental stage examined. See Tables I, 2 and 3 for transcript abbreviations.

\section{Cellular localization of differentially expressed genes}

To determine the cell type-specific expression of representative transcripts in the ovary, in situ hybridization was carried out on ovarian histological sections using specific antisense riboprobes. For these experiments, we selected transcripts that were up-regulated in vitellogenic and mature ovaries, zp3, tob1a, mapk13 and mkrn1 (Figure 6), or in atretic ovaries, apoc1, s100a10, thbs and lect2 (Figure 7 ). The $z p 3$ hybridization signal was weakly detected in the cytoplasm of previtellogenic oocytes, whereas the signal increased in early cortical alveolus stage oocytes to subsequently diminished again at later stages (Figure 6A and $6 \mathrm{~B}$ ). The staining was absent in vitellogenic oocytes as well as in the surrounding follicle cell layers. A similar localization pattern was observed for tob1a (Figure 6D and 6E) and mkrn1 (Figure 6J and 6K), although their hybridization signals remained visible, but much weaker, in the cytoplasm during vitellogenesis. A weak $m k r n 1$ staining was also seen in follicular cells of vitellogenic follicles. mapk13 transcripts were exclusively localized in the surrounding follicular cells of late vitellogenic oocytes, whereas expression in ovarian follicles at other stages was not consistently detected (Figure 6G and 6H). For all these transcripts, sense probes resulted in no signal (Figure 6C, F, I and 6L). 


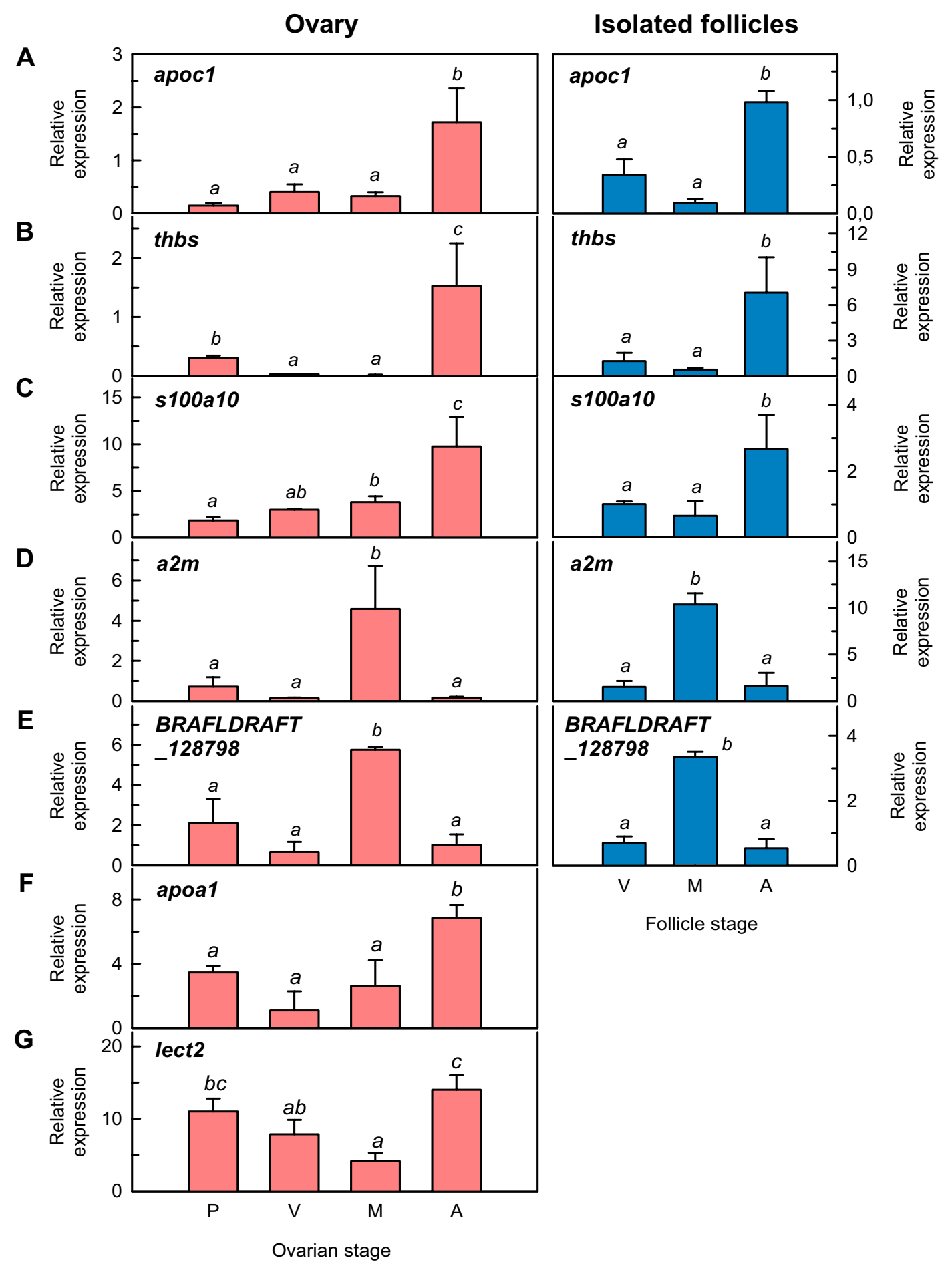

Figure 5

Expression profile of selected transcripts during Senegalese sole ovarian development. Histograms represent relative mean expression values \pm SEM ( $n=3$ females) in ovaries (left panels) or in isolated ovarian follicles (right panels) of apolipoprotein C-I (apocl; A), thrombospondin (thbs; B), SI00AIO calcium binding protein (s I00al0; C), alpha-2-macroglobulin (a2m; D), hypothetical protein BRAFLDRAFT_I28798 (BRAFLDRAFT_I28798; E), apolipoprotein A-I (apoal; F), and leukocyte cell-derived chemotaxin 2 (lect2; G). Data were determined by qPCR and normalized to I8S ribosomal protein (I8S) expression. Data with different superscript are statistically significant $(p<0.05)$. 


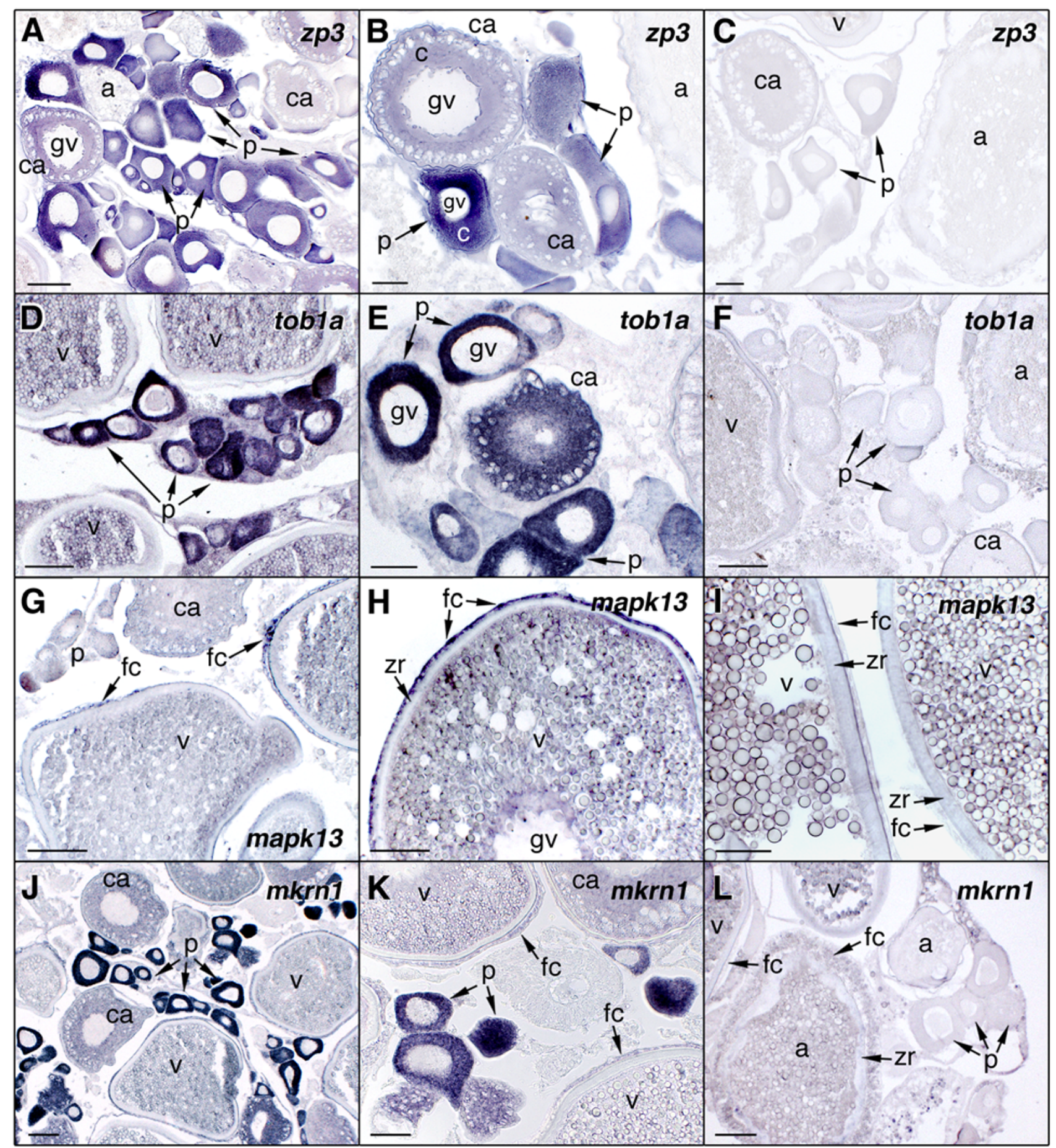

Figure 6

In situ hybridization of zona protein 3 (zp3), transducer of ERBB2 (tob/a), mitogen-activated protein kinase p38delta (mpkl3), and makorin RING zinc finger protein Ia (mkrn I) transcripts in the Senegalese sole ovary. Ovarian histological sections were hybridized with antisense digoxigenin-labeled riboprobes for $z p 3$ (A and B), tob/a (D and E), $m p k / 3(\mathrm{G}$ and $\mathrm{H})$ and $m k r n /(\mathrm{J}$ and $\mathrm{K})$. The hybridization signal is colored dark-blue to purple. No staining signal was observed using sense probes (C, F, I and L). gv, germinal vesicle; p, previtellogenic ovarian follicle; ca, ovarian follicles with oocytes at the cortical-alveolus stage; c, oocyte cytoplasm; a, atretic ovarian follicle; zr, zona radiata; fc, follicular cells. Bars, $50 \mu \mathrm{m}$ (B, C, D, E, $\mathrm{F}, \mathrm{H}, \mathrm{I}$, and $\mathrm{K}), \mathrm{I} 00 \mu \mathrm{m}(\mathrm{A}, \mathrm{G}, \mathrm{J}$ and $\mathrm{L})$. 


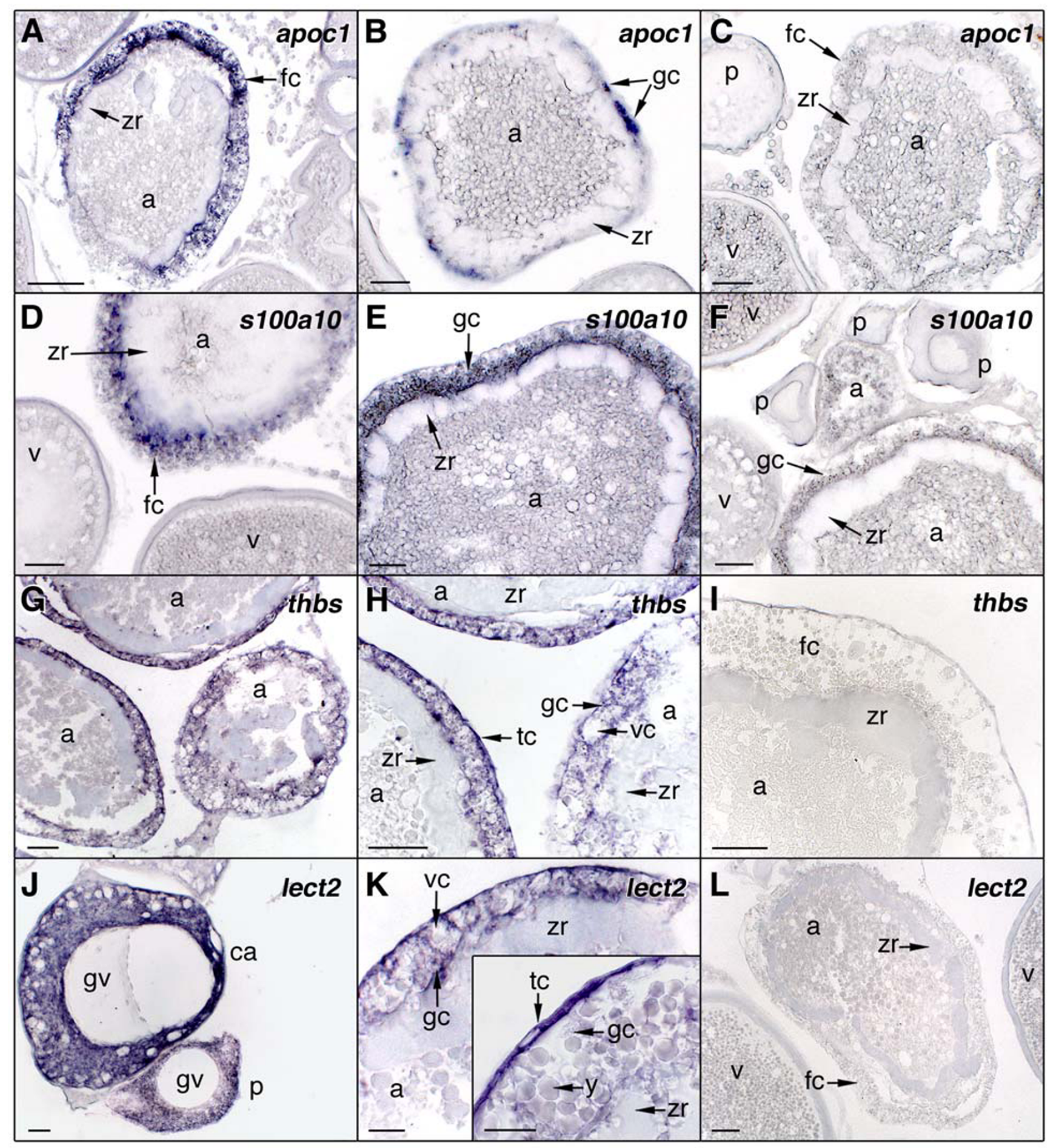

Figure 7

In situ hybridization of apolipoprotein C-I (apocl), SI 00A I 0 calcium binding protein (sl 00al0), thrombospondin (thbs), and leukocyte cell-derived chemotaxin 2 (lect2) transcripts in the Senegalese sole ovary. Ovarian histological sections were hybridized with antisense digoxigenin-labeled riboprobes for apocl (A and B), s/00al0 (D and E), thbs $(\mathrm{G}$ and $\mathrm{H})$, and lect2 $(\mathrm{J}$ and $\mathrm{K})$. No staining signal was observed using sense probes (C, F, I and $\mathrm{L})$. gc, granulosa cells; tc, theca cells; vc, vacuole in granulosa cells; y, yolk granules. Other abbreviations as in Fig. 5. Bars, $20 \mu \mathrm{m}(\mathrm{J}, \mathrm{K}), 50 \mu \mathrm{m}(\mathrm{B}, \mathrm{C}, \mathrm{D}, \mathrm{E}, \mathrm{F}, \mathrm{G}$, $\mathrm{H}, \mathrm{I}$ and $\mathrm{L}), \mathrm{I} 00 \mu \mathrm{m}(\mathrm{A})$. 
Regarding the transcripts up-regulated during ovarian atresia, apoc1-specific antisense probes showed an intense and specific staining in hypertrophied and vacuolized follicular cells of atretic follicles, which was increasing as follicular atresia progressed (Figure 7A and 7B). The same staining pattern was found for $5100 a 10$ (Figure 7D and $7 \mathrm{E}$ ) and thbs (Figure 7G and $7 \mathrm{H}$ ). The lect 2 transcripts were found in theca cells of atretic follicles (Figure 7K inset) but a weaker and more diffuse staining was also detected in hypertrophied granulosa cells (Figure 7K). Primary growth oocytes, including cortical alveolus stage oocytes, also expressed thbs and lect2 mRNAs (Figure 7J), in agreement with their increased levels previously found in previtellogenic ovaries by qPCR (Figure 5B and 5G). Sense probes for all of these transcripts were negative (Figure $7 \mathrm{C}, \mathrm{F}, \mathrm{I}$ and $7 \mathrm{~L}$ ).

\section{Discussion}

The present work has identified a number of differentially expressed genes in the Senegalese sole ovary that may play different roles during ovarian follicle growth and maturation, as well as several genes that were not previously found to be regulated in the teleost ovary. The expression of some genes specifically in follicular cells of atretic follicles suggest the role of these cells in the activation of molecular pathways associated with ovarian follicle atresia which have not been previously recognized in fish.

\section{Microarray performance}

In this study, a first-generation Senegalese sole oligonucleotide microarray was employed. This array represents the second high-density microarray available for commercial flatfish, in addition to that recently published for Atlantic halibut (Hippoglossus hippoglossus) [34], and has been previously shown to perform well to detect differences in gene expression [27].

Transcriptome analysis during ovarian growth, maturation, and follicular atresia in Senegalese sole showed the differential expression of 118 genes. This number of regulated genes is lower than that reported in similar studies on trout ovaries by using cDNA microarrays $[13,15]$ or SSH [17], where changes in the expression of up to 600 genes have been reported. However, our data are more similar to the expression profiling obtained from the comparison of halibut larval stages not very distant during development (e.g., mouth opening vs. post-hatch) by using an oligo microarray (44 differentially expressed genes in Atlantic halibut [34]). The apparent discrepancy in the overall number of genes regulated during ovarian development observed in this work with respect to published reports in salmonids using cDNA microarrays may be related to the limited number of unique genes represented in our array when compared with the salmonid platforms, or to the fact that oligo arrays are usually more stringent than cDNA arrays [35]. Another important aspect that can be considered is that salmonids have synchronous ovaries, unlike the Senegalese sole that has a group-synchronous ovary, and therefore ovarian follicles at different developmental stages are present at any time during the spawning season.

\section{Folliculogenesis and oocyte growth}

The period of ovarian vitellogenesis in fish is mainly regulated by the follicle-stimulating hormone (FSH) and involves the differentiation and growth of ovarian follicles mainly by the incorporation of circulating vitellogenins and very low-density lipoproteins (VLDL) in the oocyte [36]. Among the genes regulated in Senegalese sole vitellogenic ovaries, the GO terms overrepresented belong to the metabolic, cellular, biological and developmental processes categories, and this is consistent with the rapid rates of growth and development of the ovarian follicles at this stage. Thus, transcripts possibly related to mitochondrial energy production (cox1, cytb, nd3, nd1, acca2), cystoskeleton formation and organization (bactin1, actc1l, tagln, tpm1-1, krt8, myh11), intracellular signaling pathways ( $r h o g, s g k)$, and cell-to-cell and cell-to-matrix interactions (mibp2, thbs-4b, zgc:64051), that may play different roles during the formation and growth of the ovarian follicles, were over-expressed relative to previtellogenic ovaries. Also, as seen in previous ovarian transcriptome studies in salmonids and tilapia $[15,17,37]$, as well as in zebrafish fully-grown ovarian follicles [12], zona radiata (zp3) and $h s p 90 b$ transcripts were strongly up-regulated. Vertebrate members of the heat shock protein 90 family play a post-translational regulatory role within the cell by interacting with several important cellular signalling molecules and transcription factors, such as steroid receptors, modulating their activity [38]. High abundance of $h s p 90 b$ transcripts is a common feature of mammalian, fish and Drosophila ovaries and eggs [12].

During oocyte growth, meiosis is arrested at prophase I, and it will not proceed until it is activated by the maturation promoting (MPF). The MPF is a cytoplasmic complex specifically formed during oocyte maturation consisting of cyclin $\mathrm{B}$ and $\mathrm{Cd} 2$, a serine-threonine protein kinase [39]. Studies in zebrafish have shown that immature oocytes contain $\mathrm{Cd} 2$ proteins but not cyclin $\mathrm{B}$, and therefore the absence of cyclin $\mathrm{B}$ translation is likely the main mechanism to maintain meiosis arrest [40]. However, the roles of other cyclins (e.g., cyclin A) and protein kinases during this process in growing fish oocytes is poorly known. It is therefore of interest the strong repression of a transcript similar to vertebrate CDC-like kinase 2 (si:ch211-81a5.7) in sole vitellogenic ovaries.

The vitellogenic period is also characterized by intense deposition of RNA and proteins, as well as lipids, vitamins 
and hormones, which are necessary during the earliest steps of embryonic development [41]. Maternal RNAs are produced endogenously by the oocyte and stored during oogenesis, and they become usable for embryogenesis upon egg activation and fertilization, usually sometimes after a process of activation involving translation or protein modification [42]. One of these transcripts found in the Senegalese sole ovary, and highly expressed in previtellogenic oocytes of vitellogenic ovaries, was tob1a. Interestingly, the array and qPCR analyses detected the up-regulation of tob1a only in vitellogenic ovaries, which may suggest that this transcript starts to be accumulated in previtellogenic oocytes at the onset of vitellogenesis. tob1a is also found in trout ovaries [15] and encodes a transcriptional repressor of the BTG/Tob family of antiproliferative proteins [43]. Tob1a plays an important role during embryonic dorsoventral patterning in zebrafish by inhibiting transcriptional regulation stimulation by $\beta$-catenin, a factor that is essential for the dorsal development of amphibian and fish embryos [44]. Other transcripts that may also be stored in oocytes are nrxn1a and mkrn1. nrxn1a is a member of a family of adhesion molecules involved in the formation and function of synapses. In both zebrafish and amphibians $[45,46]$, neurexins genes are expressed in the ovary and in embryos before the activation of zygotic transcription, and interestingly, a parental origin of some of the embryonic neurexin isoforms has been suggested [45]. The mkrn1, as tob1a, was detected at high levels in Senegalese sole previtellogenic oocytes of vitellogenic ovaries. This transcript encodes a putative ribonucleoprotein with a distinctive array of zinc finger domains that may play an important role in embryonic development and neurogenesis, as reported for the amphibian makorin-2 [47].

The most highly up-regulated transcript in sole vitellogenic ovaries showed sequence similarity to tilapia selenoprotein W2a (sepw2a). Selenoproteins are a diverse group of proteins, with 25 members in humans [48], that contain selenocysteine (Sec) which is incorporated by a redefined in-frame UGA codon and requires the involvement of a complex translational machinery [49]. Selenoproteins with characterized functions are enzymes involved in redox reactions, such as glutathione peroxidase, thioredoxin reductase, and iodothyronine deiodinase, and thus they are believed to protect the cells from oxidative damage and apoptosis [49]. Expression of selenoproteins in fish ovaries has also been reported in salmonids [15] and tilapia [37]. These coincident findings suggest that seleneproteins might be accumulated in fish follicles for protection against oxidative stress during folliculogenesis and oocyte growth. In mammals, selenium stimulates proliferation of granulosa cells from small follicles and also potentiates FSH induction of estradiol secretion [50]. In addition, accumulation of maternal selenoproteins in ovarian follicles may have a role for antioxidant protection of the offspring [50].

\section{Oocyte maturation and hydration}

During the maturation of follicle-enclosed oocytes, meiosis is reinitiated in response to progestagens produced by the follicular cells after luteinizing hormone (LH) stimulation [39]. During this process, the germinal vesicle migrates towards the oocyte periphery, the nuclear envelope breaks down, the first meiotic division occurs, and the chromosomes proceed to second meiotic metaphase where they arrest; at this point, the oocyte will ovulate and becomes an egg [51]. Oocyte maturation is also accompanied by important changes in the cytoarchitecture and function of the ovarian follicle, since steroidogenic pathways in granulosa cells are switched from estrogen to progestagen production, intercellular communication oocyte-granulosa cells is resumed, and the zona radiata becomes more compacted $[39,52,53]$. At this stage, ovarian transcripts associated with the regulation of intracellular signalling pathways (such as mapk13 in follicular cells, and ywhae), and cystoskeleton (mybph, gsna, myo10, LOC100005008), as well as in the assembly of the nucleosome (hist1h2be, myst3), were significantly regulated in Senegalese sole, which is consistent with the important nuclear and cytoplasmic changes occurring in the ovarian follicle during oocyte maturation.

One transcript overexpressed in mature ovaries was $e$-syt2$a$ which is related to the synaptotagmin family of vesicle proteins that are believed to function as calcium sensors for vesicle exocytosis at synapsis [54]. It is known that fish oocytes, as the eggs of most organisms, suffer a transient elevation of intracellular free $\mathrm{Ca}^{2+}$ following fertilization, an event that triggers a series of biochemical pathways required for the block of polyspermy, activation of metabolism, re-entry into the cell cycle, and execution of the developmental program [55]. One of the earliest responses to the $\mathrm{C}^{2+}$ wave in the oocyte is the cortical alveoli exocytosis wich produces an elevation of the chorion and the separation of the egg surface [55]. Studies in mammalian eggs suggest that the release of cortical granules in mature eggs is dependent upon calcium-dependent synaptosome-associated protein 25 (SNAP-25) which might be regulated by binding to $\mathrm{Ca}^{2+}$-dependent synaptotagmins as it occurs in neurons $[56,57]$. These observations therefore suggest that the induction of $e$-syst2-a transcripts in sole mature ovaries might be part of the molecular pathways activated in the oocyte in preparation for fertilization.

In marine teleost that produce buoyant (pelagic) eggs, such as the Senegalese sole, oocytes continue to enlarge during maturation owing to hydration [58]. The hydrolysis of the oocyte yolk proteins by the lysosomal proteases 
cathepsin B (Catb) and/or cathepsin L (Catl) that occurs during maturation results in the increase of free amino acids in the ooplasm, incrementing the osmotic pressure of the oocyte, and hence facilitating water uptake mediated by aquaporin-1b (Aqp1b) [58-60]. Current evidence in some fish species suggest that both Catb and Aqp1b are regulated post-translationally rather than transcriptionally in oocytes during meiotic maturation [61-63]. Accordingly, in the present study, we did not detect changes in the expression levels of $a q p 1 b$ and catl, as well as of other cathepsins and proteases, for which specific oligos were present in the microarray. However, probes for $c a t b$ were lacking, and therefore the regulation of this transcript during oocyte maturation in Senegalese sole can not be ruled out. Nevertheless, the up-regulation of $z g c: 77650$, a transcript showing sequence similarity to a GTP-binding protein (ADP-ribosylation factor 4) involved in protein trafficking that may modulate vesicle budding and uncoating within the Golgi apparatus [64], it is of interest. This observation may indicate a role of vesicle trafficking during oocyte maturation that might be important, for instance, in the delivery of lysosomal cathepsin to yolk granules or in the control of Aqp1b shuttling into the oocyte plasma membrane. These mechanisms are however not yet elucidated in fish oocytes and need to be investigated in the future.

The accumulation of inorganic ions in oocytes undergoing maturation, mainly $\mathrm{K}^{+}$and $\mathrm{Na}^{+}$, may account for about $50 \%$ of the final osmotic pressure, and therefore it is considered as an additional mechanism mediating fish oocyte hydration [59]. Interestingly, we found that three of the most highly up-regulated transcripts in mature ovaries corresponded to $\mathrm{Na}^{+}-\mathrm{K}^{+}$-ATPase subunits (atp1b1a, $a t p 1 a 1$ and $a t p b)$, whereas the solute carrier slc34a2a, also known as the type II $\mathrm{Na}^{+} / \mathrm{Pi}$ cotransporter, was one of the most down-regulated transcripts. In trout, $\operatorname{slc} 26\left(\mathrm{Na}^{+}-\right.$ independent chloride/iodide transporter) and aquaporin4 (aqp4) were found to be overexpressed in ovarian tissue at maturation [13]. The causes for the different ion and water transport-associated transcripts regulated during oocyte maturation in sole and trout are intriguing, although the fact that trout oocytes exhibit a much lower hydration than Senegalese sole oocytes, resulting in the production of demersal eggs, may be one of the reasons.

In vertebrates, retinoic acid regulates the transcription of many genes involved in embryonic development and germ cell differentiation through binding to nuclear receptors (retinoic acid receptors, RARs and retinoid $x$ receptors, RXRs) [65]. In mammals, retinoic acid also affects the acquisition of developmental competence of oocytes and the steroidogenesis of ovarian follicle cells $[66,67]$. In fish, recent studies in trout suggest that follicular cells express several genes associated with retinoid and carotenoid metabolism indicating the presence of an additional pathway to provide retinoids to the oocyte in addition to the receptor mediated uptake of lipoproteins [68]. A transcript related to this system was induced in Senegalese sole mature ovaries, rarres 3 , which shows sequence similarity to the tazarotene (synthetic, topical retinoid)-induced gene 3 (TIG3; Retinoic Acid Receptor Responder 3 ). This gene encodes a growth regulator that possibly mediates some of the growth suppressive effects of retinoids [69]. Although the cell localization of rarres 3 in the ovarian follicle was not determined here its overexpression in the mature sole ovary may indicate that retinoids could play an additional paracrine role by affecting the expression of suppressor/growth regulatory pathways in the ovary.

Another gene that could play a paracrine role in the sole ovary at the maturation stage is the proteinase inhibitor $a 2 m$. In the mammalian ovary, $a 2 m$ modulates the actions of growth factors and cytokines, and recent works suggest that it may have autocrine or paracrine roles in granulosa cells potentially important for regulation of estradiol production and development of dominant follicles [70]. Interestingly, we observed that the expression of this mRNA was specifically up-regulated during maturation, whereas in atretic ovaries its induction was prevented. In contrast, down-regulation of $a 2 m$ was reported in trout precocious mature ovaries [15].

Finally, similarly to that described in trout ovaries at the time of meiosis resumption, we found high levels of coagulation factor II (thrombin I), $f 2$, in mature ovaries. In trout, overexpression of the coagulation factor $\mathrm{V}(c f 5)$ has been speculated to be related with the prevention of bleeding from ruptured ovarian follicles at the time of ovulation [13]. Although in Senegalese sole we found the induction of an apparently different coagulation factor, a similar scenario may be considered to occur in the flatfish ovary.

\section{Follicular atresia}

Ovarian atresia is a common phenomenon in teleosts under both natural and experimental conditions during which a number of vitellogenic ovarian follicles fail to complete maturation and ovulation, degenerate and are eventually reabsorbed $[19,20]$. Ovarian follicle atresia in fish seems not to be mediated by apoptosis in the follicular cells, unlike in mammals, and thus this process appears to be different than the post-ovulatory follicular reabsorption mechanism, which is apparently mediated by apoptosis $[22,71,72]$. Therefore, apoptosis may not be relevant at the onset of atresia, although it may contribute to a more efficient removal of atretic follicles during ovarian follicular regression after spawning $[73,74]$. The ovarian transcriptome analysis in Senegalese sole might 
support this view since the expression levels of none of the apoptosis-related genes that were represented in the microarray, identified by GO annotation, changed in atretic ovaries related to vitellogenic/mature ovaries.

In Senegalese sole, as in other teleosts $[19,20]$, the process of ovarian follicle atresia and resorption is preceded by marked morphological changes in both the oocyte and follicular cells, such as the disintegration of the oocyte germinal vesicle and of other cytoplasmic organelles, the fragmentation of the zona radiata, and the hypertrophy of the granulosa cells. These cells become phagocytic with digestive vacuoles and incorporate and digest the oocyte yolk as well as other oocyte components and organelles, and they may also secrete enzymes which digest the yolk $[19,20,73,75]$. In atretic ovaries, two of the up-regulated genes corresponded to apoa1 and apoc1, which are part of chylomicrons, very low density lipoproteins (VLDL) and high density lipoproteins (HDL) involved in lipid transport in the bloodstream [76]. In addition, we found reduced elovl1b transcripts possibly involved in the control of the synthesis of very long chain fatty acids and sphingolipids in the ovary [77]. Studies in rainbow trout have shown that during the course of follicular atresia there is a massive transfer of the oocyte yolk proteins, and possibly lipids, into the bloodstream combined with HDL [78], as a result of the ingestion and digestion of the yolk by the follicular cells $[19,20]$. The finding of high apoa1 and apoc 1 transcript levels in hypertrophied follicular cells (at least for apoc1) of Senegalese sole, together with that recently reported for the fatty acid-binding protein 11 (fap11) [79], provides additional evidence for this mechanism in teleosts. However, the nature of the numerous invasive apoc1 - and fabp11-expressing cells (theca or granulosa cells) remains to be clearly established. Nevertheless, these data suggest the importance of lipid-metabolic processes during follicular atresia in fish [79], which may have evolved to facilitate the redistribution of energy-rich yolk materials from oocytes that fail to develop properly [80].

In humans, Apoc-I is primarily expressed in the liver but also in the lung, skin, spleen, adipose tissue, and brain [81]. ApoC-I can interact with lipid surfaces and play an important role in controlling plasma lipoprotein metabolism by the regulation of several enzymes, such as lipoprotein lipase or phospholipase A2 [76]. The expression of $A p o C-I$ in the mammalian ovary has not been reported, unlike that of $A p o A-I$ and $A p o E$ which are expressed by luteinizing granulosa cells and theca cells, respectively, of atretic follicles [82,83]. Intraovarian ApoE controls theca cell production of androgens as well as limiting the size of the theca cell compartment [83]. Teleost ovary and embryos also express an ApoC-I ortholog as it has been recently shown $[18,84,85]$ and confirmed in the present study, although its function is largely unknown. In the embryo, apoc1 is localized in the yolk syncytial layer [85], along with apoe, apoa1 and apo14 [86,87], suggesting its role in the nutrition of the developing embryo through the synthesis and secretion of apolipoproteins and lipoproteins. Therefore, the expression of apoc1 in follicular cells of fish atretic follicles, which has not been previously reported, may have a similar role for the resorbption of lipids and lipoproteins stored in the oocyte. Interestingly, trout eggs obtained by hormonal induction, which result in alevins with a high percentage of morphological abnormalities at the yolk-sac resorption stage, also show a dramatic increase of apoc1 [18]. Altogether, these findings suggest that ApoC-I could be a useful marker to identify factors involved in premature ovarian regression and abnormal embryo development in cultured fish. It is worth noting that in humans it has been recently proposed that serum ApoC-I may be useful for early demonstration of metabolic abnormality in women with polycystic ovary syndrome [88].

In histological sections of Senegalese sole ovarian follicles at advanced atresia, we observed the presence of blood cells such as erythrocytes and leukocytes, possibly derived from the ovarian stroma and/or the theca, which invaded the degenerating oocyte. The presence of granulocytes (polymorphonuclear leukocytes) in atretic follicles is reported in other fish species $[21,89,90]$, and suggest a relationship between follicular regression and immune cells [73]. The specific function of immune cells (eosinophilic granulocytes and macrophages) during follicular atresia in fish is not well known, although it has been proposed that they may act synergistically with follicular cells in the resorption of the oocyte by releasing their granules containing lytic enzymes [89]. In the mammalian ovary, the leukocyte-ovarian cell interactions through the release of chemokines is believed to play an important role for leukocyte recruitment and activation during follicular atresia, ovulation and luteal function [91]. In teleosts, however, the molecular mechanisms mediating the invasion of immune cells in atretic follicles are largely unknown. In the present study, we noted high lect 2 expression levels in theca cells of atretic follicles, a transcript related to mammalian Lect 2 which encodes a protein with chemotactic properties for human neutrophils [92]. This observation may provide evidence for the presence of a chemotaxin-mediated mechanism for leukocyte accumulation in fish follicles at advanced atresia, similarly to that occurs during the formation of the corpus luteum in the mammalian ovary [91]. However, whereas a number of different chemokines have been found in the mammalian ovary [91], ovarian expression of Lect2 has not been yet reported, and therefore the structural and functional relationships of Senegalese sole lect2 with other ovarian chemokines requires further investigation. 
In addition to lect2, we also found high expression levels of the calcium binding protein-encoding gene s100a10 in atretic follicles. In mammals, S100 proteins are localized in the cytoplasm and/or nucleus of a wide range of cells and are involved in the regulation of a number of cellular processes such as cell cycle progression and differentiation [93]. In the ovary, S100a10 plays an antiapoptotic function by binding the $\mathrm{Bcl}-\mathrm{xL} / \mathrm{Bcl}-2$-associated death promoter and its expression in granulosa cells is stimulated by gonadotropins and follicle survival factors, including the epidermal growth factor, the basic fibroblast growth factor, and interleukin-1 $\beta$ [94]. It is known, however, that some S100 proteins can also be released into the extracellular space and act as chemoattractants for leukocytes or activators of macrophages [93]. Therefore, the high expression levels of s100a10 in sole atretic follicles may play a dual function, to protect follicular cells from apoptosis during atresia and to act as chemoattractant for leukocytes and macrophages. In support of this hypothesis is the reported down-regulation of an S100 homologue in trout post-ovulatory follicles [95], which based on our phylogenetic analysis is in fact an $\$ 100 a 10$ ortholog.

The mammalian ovary is distinctive in that it is a tissue that undergoes physiological angiogenesis, in which blood vessels are programmed to develop and regress in a cyclic manner [96]. This mechanism is tightly regulated by pro- and antiangiogenic factors such as the members of the thrombospondin (TSP) family TSP-1 and TSP-2, which are among the naturally occurring inhibitors of angiogenesis. These secreted proteins are expressed by granulosa cells of atretic follicles and in the corpus luteum after ovulation in rats and primates [96,97], suggesting that they may be involved in the cessation of angiogenesis in follicles undergoing atresia [96]. Our microarray analysis revealed that a thrombospondin isoform (thbs), distinct from $t h b s 4 b$ which was overexpressed in vitellogenic ovaries, was up-regulated in Senegalese sole follicular cells during follicular atresia. thbs was similar to mammalian TSP-1 and TSP-2, which indicates that the inhibition of angiogenesis may be an important mechanism regulating atresia in both mammalian and fish ovarian follicles. However, primary growth oocytes also expressed thbs which may point to an additional role of this protein during folliculogenesis and/or as a maternal molecule for early embryonic development.

In contrast to apoptosis, Wood and Van Der Kraak [22,80] proposed that proteolytic degradation of yolk proteins, mediated by the differential activation of Catl in the oocyte, may be the initial event leading to follicular atresia in fish. In the present study we did not detect changes in the expression of catl transcripts in atretic ovaries, which may suggest a potential post-transcriptional regulation of this protease during ovarian follicle atresia as discussed earlier. In any event, the specific oocyte mechanisms involved in the regulation of protease activity during atresia in fish, as well as the origin of the signals that presumably activate this system, are still largely unknown and warrant further investigation.

\section{Conclusion}

The present study has contributed to identify differences in gene expression during ovarian development in Senegalese sole despite that, at this point, the microarray platform employed was not ovary-specific and contained a limited number of represented genes. Some of the genes identified were not described before in the teleost ovary, and therefore the present data provide a basis for future studies on their regulation and function. Particularly, determination of the cell-type specific localization of some transcripts suggest the involvement of follicular cells in yolk resorbption, chemotaxin-mediated leukocyte migration, angiogenesis and prevention of apoptosis during ovarian atresia. These observations also indicate that some of the transcripts, such as e-syt2-a, apoc1 and s100a10, may be useful markers contributing to the identification of factors involved in the acquisition of egg fertilization competence and follicular regression. Clearly, however, further experimental studies will be necessary to determine when these mRNAs are translated as well as the physiological functions of the encoded proteins. The future increase in the sequencing of the Senegalese sole ovarian transcriptome will enhance our knowledge on the molecular pathways involved in oogenesis, and will facilitate the comparative genomic analysis of gonad development in teleosts.

\section{Methods}

\section{Animals and biological samples}

Adult Senegalese sole $(1219 \pm 90 \mathrm{~g}) \mathrm{F} 1$ generation were maintained as previously described [24]. Females $(n=3-$ 5 ) were sacrificed at different times during the annual reproductive cycle, corresponding to different folliculogenesis stages $[24,28]$. Thus, females with previtellogenic and vitellogenic ovaries were collected during July and November, respectively. Maturing and mature ovaries were collected from females treated with intramuscular injection of $5 \mu \mathrm{g} / \mathrm{kg}$ GnRHa and killed 24-48 h later as described [24]. Finally, atretic ovaries were obtained in April-May, as well as from females treated with GnRHa. At all sampling times, fish were sedated with 500 ppm phenoxyethanol, killed by decapitation, and the body and gonads of each animal weighed to calculate the GSI (gonad weight/body weight $\times 100$ ). Pieces of the ovaries were immediately removed and placed in Petri dishes containing $10 \mathrm{ml}$ of $75 \%$ Leibovitz L-15 medium with Lglutamine (Sigma) and $100 \mathrm{mg}$ gentamicin $/ \mathrm{ml}, \mathrm{pH} 7.5$ [60]. Follicle-enclosed oocytes at vitellogenesis, maturation and atresia were manually isolated from the rest of the ovary using watchmaker forceps. One piece of the ovary, as well as the isolated ovarian follicles, were deep- 
frozen in liquid nitrogen and stored at $-80^{\circ} \mathrm{C}$ until RNA extraction. Two additional pieces of the gonad, adjacent to the piece sampled for RNA extraction, were fixed in modified Bouin solution (75\% picric acid and 25\% formalin) for histological analysis, or in $4 \%$ paraformaldehyde (PFA) for in situ hybridization. Procedures relating to the care and use of animals were approved by the Ethics Committee from Institut de Recerca i Tecnologia Agroalimentàries (IRTA, Spain) in accordance with the Guiding Principles for the Care and Use of Laboratory Animals.

\section{Histological analysis}

Ovaries fixed in Bouin solution for 3-4 h were dehydrated, embedded in paraplast, sectioned at $5 \mu \mathrm{m}$, and stained with hematoxilin-eosin. Alternatively, fixed gonads were embedded in glycol methacrylate resin (Technovit 7100, Heraeus Kultzer), sectioned at $3 \mu \mathrm{m}$ and stained with methylene blue/azure II/basic fuchsin. The percentages of previtellogenic, early vitellogenic, vitellogenic, mature and atretic oocytes were calculated by counting 100-150 total ovarian follicles in at least three different histological sections from the same ovary, as previously described [24].

\section{RNA extraction, microarray hybridization and analysis}

Total RNA was extracted from ovaries at previtellogenesis, vitellogenesis, maturation and atresia, determined by histological examination, using the RNeasy extraction kit (Qiagen) and treated with DNAse following the manufacturer's instructions. For each ovarian developmental stage, RNA was extracted from the ovary of three different females. The quality and concentration of the RNA was analyzed using the Agilent 2100 bioanalyzer and NanoDrop $^{\mathrm{TM}}$ ND-1000 (Thermo Scientific). Samples with RNA integrity number (RIN; [98]) $<6.0$ were discarded. Total RNA $(0.5 \mu \mathrm{g})$ from each of the twelve samples was amplified and labelled with fluorescent cyanine dyes, $\mathrm{Cy} 3$ or Cy5, using the Eberwein mRNA amplification procedure [99] employing the MessageAmp ${ }^{\mathrm{TM}}$ aRNA amplification kit from Ambion (Applied Biosystems) following the manufacturer's instructions with minor modifications. The Cy3and Cy5-labelled aRNAs synthesized from RNAs originated from two different ovarian stages were mixed in equal amounts and hybridized to an oligonucleotide microarray representing 5,087 Senegalese sole unigenes [27]. These 60-mer probes were designed against the 3 'end sequences of Senegalese sole expressed sequence tags (ESTs) [27]. Each hybridization was done in triplicate with aRNA from three different females, so three different biological replicates per ovarian stage were analyzed. To estimate the rate of false-positive expression, a self-to-self hybridization was carried out, in which total RNA from two different aliquots of previtellogenic ovaries were used to produce either Cy3 or Cy5 labelled aRNA. Hybridizations were carried out for $17 \mathrm{~h}$ at $60^{\circ} \mathrm{C}$ using Agilent's gaskets G2534-60002, G2534A hybridization chambers, and
DNA Hybridization Oven G2545A, according to the manufacturer's instructions.

Microarray raw data were obtained using Agilent's DNA Microarray Scanner G2505B and Feature Extraction software (v10.1). The raw fluorescence intensity data were processed using the Polyphemus ${ }^{\mathrm{TM}}$ software (Oryzon Genomics), which includes spatial data compensation, non-significant expressed data filtering, and data normalization. Data normalization was carried out by an improved version of the nonlinear Q-splines normalization method [100], enhanced with robust regression techniques. Normalized and log-transformed data were used to calculate the FC values. Differential expression was assessed with Polyphemus ${ }^{\mathrm{TM}}$ analyzing biological replicates based on repeated experiments using robust statistics on average technical replicates removing the outlier points (caused by dust or array imperfection). The p-values were calculated based on the absolute value of the regularized t-statistic [101], which uses a Bayesian framework to derive the algorithm, using internal replicated controls to assess the minimum technical variability of the process. A $p$-value $<0.01$ was considered significant. Cut-offs for significant changes were always greater than the inherent experimental variation as assessed by the FC of internal controls and/or self-to-self hybridizations. The microarray data have been deposited in NCBI's [102] Gene Expression Omnibus (GEO) [103] and are accessible through GEO Series accession number GSE17337 [104].

\section{EST Annotation, gene ontology and amino acid sequence analysis}

Annotation of ESTs and extraction of GO terms to each obtained hit using existing annotations was done using the BLAST2GO v1 program [31] as previously described [27]. ESTs that were considered significantly expressed after microarray analysis, but that they could not be initially annotated, were sequenced from the $5^{\prime}$ and 3 ' ends using an ABI PRISM 377 DNA analyzer (Applied Biosystems). Basic local alignment search tool (BLAST) software [102], employing the 5'end nucleotide sequences or the consensus $5^{\prime}$ and 3 ' end sequence, was used to re-annotate these ESTs. For some of these ESTs, further annotation required the search for conserved motifs using PROSITE [105] and multiple amino acid sequence alignments using ClustalW [106]. The neighbor-joining (NJ) phylogenetic analysis [107] of the amino acid alignments was also carried out based on mean character distances. The robustness of the phylogenetic tree was tested by bootstrap analysis [108] with 1000 repetitions.

\section{Real-time quantitative RT-PCR}

The relative mRNA levels of selected ESTs during ovarian development were determined by qPCR on the same RNA samples than those employed for the microarray. Total RNA from the ovary was extracted with the RNeasy Mini kit (Qia- 
gen), and treated with DNase I using the RNase-Free DNase kit (Qiagen). An aliquot of the RNA $(0.5 \mu \mathrm{g})$ was reversetranscribed using 20 IU of AMV RT (Stratagene), $0.5 \mu \mathrm{M}$ oligo(dT) ${ }_{12-18}$ (Invitrogen) and $1 \mathrm{mM}$ dNTPs for $1.5 \mathrm{~h}$ at $50^{\circ} \mathrm{C}$. Real-time qPCR amplifications were performed in a final volume of $20 \mu$ l with $10 \mu$ SYBR $^{\circledR}$ Green qPCR master mix (Applied Biosystems), $2 \mu$ diluted (1:5) cDNA, and 0.5 $\mu \mathrm{M}$ of each primer (see Additional file 4 ). The sequences were amplified in duplicate for each sample on 384-well plates using the ABI PRISM 7900HT sequence detection system (Applied Biosystems). The amplification protocol was as follows: an initial denaturation and activation step at $50^{\circ} \mathrm{C}$ for $2 \mathrm{~min}$, and $95^{\circ} \mathrm{C}$ for $10 \mathrm{~min}$, followed by 40 cycles of $95^{\circ} \mathrm{C}$ for $15 \mathrm{~s}$ and $63^{\circ} \mathrm{C}$ for $1 \mathrm{~min}$. After the amplification phase, a temperature-determinating dissociation step was carried out at $95^{\circ} \mathrm{C}$ for $15 \mathrm{~s}, 60^{\circ} \mathrm{C}$ for $15 \mathrm{~s}$, and $95^{\circ} \mathrm{C}$ for 15 s. For normalization of cDNA loading, all samples were run in parallel using $18 \mathrm{~S}$ ribosomal protein (18S) as reference gene, since its expression between experimental samples did not show significant differences (data not shown). Negative control samples were also run in which the template was not added. To estimate efficiencies, a standard curve was generated for each primer pair from 10-fold serial dilutions (from 100 to $0.01 \mathrm{ng}$ ) of a pool of first-stranded cDNA template from all samples. Standard curves represented the cycle threshold $(\mathrm{Ct})$ value as a function of the logarithm of the number of copies generated, defined arbitrarily as one copy for the most diluted standard. All calibration curves exhibited correlation coefficients higher than 0.97 , and the corresponding real-time PCR efficiencies were above 99\%. Values of relative expression in ovaries at different developmental stage were statistically analyzed by one-way ANOVA. The significance level was set at 0.05 .

\section{In situ hybridization}

Samples of ovaries were fixed in $4 \%$ paraformaldehyde for 16-20 h at $4^{\circ} \mathrm{C}$, and subsequently dehydrated and embedded in Paraplast (Sigma). In situ hybridization on $7-\mu \mathrm{m}$ sections was carried out with digoxigenin-alkaline phosphatase (DIG-AP) incorporated cRNA probes as previously described [109]. DIG-AP riboprobes were synthesized with T3 and T7 RNA polymerases using the DIG RNA Labeling Kit (Roche). The DIG-labeled probes were detected as previously described [105], and the resulting dark blue to purple color indicated localization of the transcripts. Sections were examined and photographed with a Leica DMLB light microscope.

\section{Authors' contributions}

ATS performed RNA sample preparation and quality control for microarray hibridization, real-time PCR, bioinformatic analyses together with JL, and drafted the manuscript. MJA and EA led the hormonal induction experiments and morphometric data analysis, and carried out the histological studies. FC performed the in situ hybridization experiments. JC conceived and coordinated the study, participated in the design of the experiments and analyses of data, and was in charge of writing the final version of the manuscript. All authors read and approved the final manuscript.

\section{Additional material}

\section{Additional file 1}

Microarray hybridizations. Scatter plot of the signal intensities in the Cy3 and Cy5 channel of replica analysis of self-to-self and differential gene expression experiments (vitellogenic vs. previtellogenic ovaries, mature vs. vitellogenic ovaries, and atretic vs. vitellogenic/mature ovaries). In each scatter plot, the green and blue lines parallel to the black diagonal line represent the $\pm 3 \sigma$ limits of the data from control and Solea senegalensis specific oligos, respectively. The histograms of the distribution of fold changes (FC) as $\log 2(\mathrm{~S} / \mathrm{C})$ for control and S. senegalensis specific oligos in each experiment are shown on the right. In these panels, the green and blue curves represent the $\pm 3 \sigma$ limits on the data from control and S. senegalensis specific oligos, respectively.

Click here for file

[http://www.biomedcentral.com/content/supplementary/14712164-10-434-S1.pdf]

\section{Additional file 2}

Identification of clone pgsP0015C05 (GenBank accession number FF286365) as Senegalese sole apolipoprotein C-I (ApoC-I). (A) Amino acid sequence alignment of human, zebrafish and trout ApoC-I with Senegalese sole FF286365 and sea bream AAT45249 (putative ApoC-I). In the upper line, the protein family (pfam) database domain no. 04691 (ApoC-I) is shown in bold letters. In red color, the consensus (cons) sequence between pfam04691 and each of the amino acid sequences is indicated. The asterisks at the bottom indicate fully-conserved residues. (B) Fifty percent majority-rule bootstrap consensus phylogenetic tree of teleost ApoC-I reconstructed with the NJ method (1000 replications) based on a mean (uncorrected) character distance matrix. Only nodes with $>50 \%$ bootstrap support are indicated. The GenBank accession number of the amino acid sequences is indicated for each species. Scale bar indicates the number of amino acid substitution per site. Click here for file

[http://www.biomedcentral.com/content/supplementary/14712164-10-434-S2.pdf]

\section{Additional file 3}

Amino acid sequence analysis of Senegalese sole $\$ 100$ calcium-binding protein. (A) Amino acid alignment of sole S100 with S100 proteins of the A1, A10 and B subgroups from human and other teleosts. In the upper part is indicated the typical structure of mammalian $S 100$ proteins formed by four helices (Helix I-IV) separated by a S100 EF hand calciumbinding domain, a hinge and a canonical EF hand calcium-binding domain. Conserved residues in most of the sequences are shaded in bold, identical residues are indicated by asterisks at the bottom, and conserved amino acid substitutions and substitutions with similar amino acids are indicated by double or single dots, respectively. Conserved residues in most of the members of the S100A10 subgroup are shaded in magenta. (B) Phylogenetic tree of human and teleost $S 100$ proteins reconstructed with the NJ method (1000 replications) based on a mean (uncorrected) character distance matrix. Only sequences belonging to the A1, A10 and B subgroups are employed for the analysis. Nodes with $>60 \%$ bootstrap support are indicated. The GenBank accession number of the amino acid sequences is indicated for each species. Scale bar indicates the number of amino acid substitution per site.

Click here for file

[http://www.biomedcentral.com/content/supplementary/14712164-10-434-S3.pdf] 


\section{Additional file 4}

Nucleotide sequence of the primers employed for real-time quantitative RT-PCR. The table lists the forward and reverse oligonucleotide primers employed for $q P C R$.

Click here for file

[http://www.biomedcentral.com/content/supplementary/14712164-10-434-S4.pdf]

\section{Acknowledgements}

We thank staff of Oryzon Genomics (Dr. Elisabet Rosell, Dr. Tamara Maes, Olga Durany and Francesc Subirada) for their assistance during microarray hybridization and bioinformatic analysis. This work was supported by Genome Spain and Genome Canada within the framework of the international consortium Pleurogene ${ }^{\mathrm{TM}}$ coordinated by JC. MJA was supported by a predoctoral fellowship from the Instituto Nacional de Investigación y Tecnología Agraria y Alimentaria (INIA, Spain), and by a postdoctoral fellowship from Juan de la Cierva Programme (Spanish Ministry of Education and Science).

\section{References}

I. De Vlaming VL: Environmental control of teleost reproductive cycles: a brief review. J Fish Biol 1972, 4:131-140.

2. Kulczykowska E: A review of the multifunctional hormone melatonin and a new hypothesis involving osmoregulation. Rev Fish Biol Fish 2001, I I:321-330.

3. Izquierdo MS, Fernández-Palacios H, Tacon AG]: Effect of broodstock nutrition on reproductive performance of fish. Aquaculture 200I, 1 97:25-42.

4. Schreck CB, Contreras-Sanchez W, Fitzpatrick MS: Effects of stress on fish reproduction, gamete quality, and progeny. Aquaculture 200I, 197:3-24.

5. Sumpter JP: Environmental control of fish reproduction: a different perspective. Fish Physiol Biochem 1997, 17:25-3I.

6. Arukwe W: Cellular and molecular responses to endocrinemodulators and the impact on fish reproduction. Mar Pollut Bull 200I, 42:643-655.

7. Cerdà J, Bobe J, Babin PJ, Admon A, Lubzens E: Functional genomics and proteomic approaches for the study of gamete formation and viability in farmed finfish. Rev Fish Sci 2008, 16:54-70.

8. Li Y, Chia JM, Bartfai R, Christoffels A, Yue GH, Ding K, Ho MY, Hill JA, Stupka E, Orban L: Comparative analysis of the testis and ovary transcriptomes in zebrafish by combining experimental and computational tools. Comp Funct Genomics 2004, 5:403-418.

9. Santos EM, Workman VL, Paull GC, Filby AL, Van Look KJ, Kille P, Tyler CR: Molecular basis of sex and reproductive status in breeding zebrafish. Physiol Genomics 2007, 30: III-122.

10. Sreenivasan R, Cai M, Bartfai R, Wang X, Christoffels A, Orban L: Transcriptomic analyses reveal novel genes with sexually dimorphic expression in the zebrafish gonad and brain. PLOS ONE 2008, 3:el79I.

II. Zeng S, Gong Z: Expressed sequence tag analysis of expression profiles of zebrafish testis and ovary. Gene 2002, 294:45-53.

12. Knoll-Gellida A, André M, Gattegno T, Forgue J, Admon A, Babin PJ: Molecular phenotype of zebrafish ovarian follicle by serial analysis of gene expression and proteomic profiling, and comparison with the transcriptomes of other animals. BMC Genomics 2006, 7:46.

13. Bobe J, Montfort J, Nguyen T, Fostier A: Identification of new participants in the rainbow trout (Oncorhynchus mykiss) oocyte maturation and ovulation processes using CDNA microarrays. Reprod Biol Endocrinol 2006, 4:39.

14. MacKenzie S, Montserrat N, Mas M, Acerete L, Tort L, Krasnov A, Goetz FW, Planas JV: Bacterial lipopolysaccharide induces apoptosis in the trout ovary. Reprod Biol Endocrinol 2006, 4:46.

15. von Schalburg KR, Rise ML, Brown GD, Davidson WS, Koop BF: A comprehensive survey of the genes involved in maturation and development of the rainbow trout ovary. Biol Reprod 2005, 72:687-699.

16. von Schalburg KR, Cooper GA, Yazawa R, Davidson WS, Koop BF: Microarray analysis reveals differences in expression of cell surface and extracellular matrix components during development of the trout ovary and testis. Comp Biochem Physiol Part D: Genomics and Proteomics 2008, 3:78-90.

17. Luckenbach JA, lliev DB, Goetz FW, Swanson P: Identification of differentially expressed ovarian genes during primary and early secondary oocyte growth in coho salmon, Oncorhynchus kisutch. Reprod Biol Endocrinol 2008, 6:2.

18. Bonnet E, Fostier A, Bobe J: Microarray-based analysis of fish egg quality after natural or controlled ovulation. BMC Genomics 2007, 8:55.

19. Saidapur SK: Follicular atresia in the ovaries of nonmammalian vertebrates. Int Rev Cytol 1978, 54:225-244.

20. Guraya SS: The cell and molecular biology of fish oogenesis. In Monographs in Developmental Biology Volume 18. Edited by: Sauer HW. Karger, Basel; 1986:169-180.

21. Miranda CL, Bazzoli N, Rizzo E, Sato Y: Ovarian follicular atresia in two teleost species: a histological and ultrastructural study. Tissue Cell I999, 3 I:480-488.

22. Wood AW, Kraak G] Van Der: Apoptosis and ovarian function: novel perspectives from the teleosts. Biol Reprod 200I, 64:264-27I.

23. Imsland AK, Foss A, Conceiçao LEC, Dinis MT, Delbare D, Schram E, Kamstra A, Rema P, White P: A review of the culture potential of Solea solea and S. senegalensis. Rev Fish Biol Fish 2003, I 3:379-407.

24. Agulleiro MJ, Anguis V, Cañavate JP, Martínez-Rodríguez G, Mylonas CC. Cerdà J: Induction of spawning of captive-reared Senegal sole (Solea senegalensis) using different administration methods for gonadotropin-releasing hormone agonist. Aquaculture 2006, 257:5। I-524.

25. García-López A, Anguis V, Couto E, Canario AVM, Cañavate JP, Sarasquete C, Martínez-Rodríguez G: Non-invasive assessment of reproductive status and cycle of sex steroid levels in a captive wild broodstock of Senegalese sole Solea senegalensis (Kaup). Aquaculture 2006, 254:583-593.

26. Anguis V, Cañavate JP: Spawning of captive Senegal sole (Solea senegalensis) under a naturally fluctuating temperature regime. Aquaculture 2005, 243:133-145.

27. Cerdà J, Mercadé J, Lozano JJ, Manchado M, Tingaud-Sequeira A, Astola A, Infante C, Halm S, Viñas J, Castellana B, Asensio E, Cañavate P, Martínez-Rodríguez G, Piferrer F, Planas JV, Prat F, Yúfera M, Durany O, Subirada F, Rosell E, Maes T: Genomic resources for a commercial flatfish, the Senegalese sole (Solea senegalensis): EST sequencing, oligo microarray design, and development of the Soleamold bioinformatic platform. BMC Genomics 2008, 9:508.

28. García-López A, Couto E, Canario AVM, Sarasquete C, MartínezRodríguez G: Ovarian development and plasma sex steroid levels in cultured female Senegalese sole Solea senegalensis. Comp Biochem Physiol A Mol Integr Physiol 2007, I 46:342-354.

29. Guzmán JM, Norberg B, Ramos J, Mylonas CC, Mañanós EL: Vitellogenin, steroid plasma levels and spawning performance of cultured female Senegalese sole (Solea senegalensis). Gen Comp Endocrinol 2008, 156:285-297.

30. Wallace RA, Selman K: Cellular and dynamic aspects of oocyte growth in teleosts. Amer Zool 198I, 21:325-343.

31. Conesa A, Gotz S, García-Gómez JM, Terol J, Talon M, Robles M: Blast2GO: a universal tool for annotation, visualization and analysis in functional genomics research. Bioinformatics 2005 , 21:3674-3676.

32. Zahurak M, Parmigiani G, Yu W, Scharpf RB, Berman D, Schaeffer E, Shabbeer S, Cope L: Pre-processing Agilent microarray data. BMC Bioinformatics 2007, 8:142.

33. Morey JS, Ryan JC, VanDolah FM: Microarray validation: factors influencing correlation between oligonucleotide microarrays and real-time PCR. Biol Proced Online 2006, 8: 175- 193.

34. Douglas SE, Knickle LC, Williams J, Flight RM, Reith ME: A first generation Atlantic halibut Hippoglossus hippoglossus (L.) microarray: application to developmental studies. J Fish Biol 2008, 72:2391-2406.

35. Brennan C, Zhang Y, Leo C, Feng B, Cauwels C, Aguirre AJ, Kim M, Protopopor A, Chin L: High-resolution global profiling of 
genomic alterations with long oligonucleotide microarray. Cancer Res 2004, 64:4744-4748.

36. Babin P, Carnevali O, Lubzens E, Schneider WJ: Molecular aspects of oocyte vitellogenesis in fish. In The fish oocyte: from basic studies to biotechnological applications Edited by: Babin PJ, Cerdà J, Lubzens E. Springer, The Netherlands; 2007:39-76.

37. Chu S-L, Weng C-F, Hsiao C-D, Hwang P-P, Chen Y-C, Ho J-M, Lee $S$-j: Profile analysis of expressed sequence tags derived from the ovary of tilapia, Oreochromis mossambicus. Aquaculture 2006, 25 I:537-548.

38. Krone PH, Sass JB, Lele Z: Heat shock protein gene expression during embryonic development of the zebrafish. Cell Mol Life Sci 1997, 53:122-129.

39. Nagahama $Y$, Yamashita M: Regulation of oocyte maturation in fish. Dev Growth Differ 2008, 50(SuppI I):SI95-S219.

40. Kondo T, Yanagawa T, Yoshida N, Yamashita M: Introduction of cyclin $B$ induces activation of the maturation-promoting factor and breakdown of germinal vesicle in growing zebrafish oocytes unresponsive to the maturation-inducing hormone. Dev Biol 1997, 190: I42-152.

4I. Tyler CR, Sumpter JP: Oocyte growth and development in teleosts. Rev Fish Biol Fish 1996, 6:287-318.

42. Lyman-Gingerich J, Pelegri F: Maternal factors in fish oogenesis and embryonic development. In The fish oocyte: from basic studies to biotechnological applications Edited by: Babin PJ, Cerdà J, Lubzens E. Springer, The Netherlands; 2007: I4I-I74.

43. Jia S, Meng A: Tob genes in development and homeostasis. Dev Dyn 2007, 236:913-921.

44. De Robertis EM, Kuroda $\mathrm{H}$ : Dorsal-ventral patterning and neural induction in Xenopus embryos. Annu Rev Cell Dev Biol 2004, 20:285-308.

45. Rissone A, Monopoli M, Beltrame M, Bussolino F, Cotelli F, Arese M: Comparative genome analysis of the neurexin gene family in Danio rerio: insights into their functions and evolution. Mol Biol Evol 2007, 24:236-252.

46. Zeng Z, Sharpe CR, Simons JP, Górecki DC: The expression and alternative splicing of alpha-neurexins during Xenopus development. Int J Dev Biol 2006, 50:39-46.

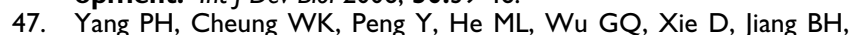
Huang $\mathrm{QH}$, Chen Z, Lin MC, Kung HF: Makorin-2 is a neurogenesis inhibitor downstream of phosphatidylinositol 3-kinase/ Akt (PI3K/Akt) signal. J Biol Chem 2008, 283:8486-8495.

48. Kryukov GV, Castellano S, Novoselov SV, Lobanov AV, Zehtab O, Guigó R, Gladyshev VN: Characterization of mammalian selenoproteomes. Science 2003, 300:|439-1443.

49. Lu J, Holmgren A: Selenoproteins. J Biol Chem 2009, 284:723-727.

50. Pappas AC, Zoidis E, Surai PF, Zervas G: Selenoproteins and maternal nutrition. Comp Biochem Physiol B Biochem Mol Biol 2008, 15 1:361-372.

51. Selman K, Wallace RA: Cellular aspects of oocyte growth in teleosts. Zool Sci 1989, 6:2II-23I.

52. Cerdà J, Reidenbach S, Prätzel S, Franke WW: Cadherin-catenin complexes during zebrafish oogenesis: heterotypic junctions between oocytes and follicle cells. Biol Reprod 1999, 6 I :692-704.

53. Yamamoto $Y$, Yoshizaki G: Heterologous gap junctions between granulosa cells and oocytes in ayu (Plecoglossus altivelis): formation and role during luteinizing hormone-dependent acquisition of oocyte maturational competence. J Reprod Dev 2008, 54: I-5.

54. Yoshihara M, Montana ES: The synaptotagmins: calcium sensors for vesicular trafficking. Neuroscientist 2004, 10:566-574.

55. Kinsey WH, Sharma D, Kinsey SC: Fertilization and egg activation in fishes. In The fish oocyte: from basic studies to biotechnological applications Edited by: Babin PJ, Cerdà J, Lubzens E. Springer, The Netherlands; 2007:397-409.

56. Zhang X, Kim-Miller MJ, Fukuda M, Knowalchyk JA, Martin TF: $\mathbf{C a}^{2+}$ dependent synaptotagmin binding to SNAP-25 is essential for $\mathrm{Ca}^{2+}$-triggered exocytosis. Neuron 2002, 34:599-6II.

57. Sun QY: Cellular and molecular mechanisms leading to cortical reaction and polyspermy block in mammalian eggs. Microsc Res Tech 2003, 6 I:342-348.

58. Cerdà J, Fabra M, Raldúa D: Physiological and molecular basis of fish oocyte hydration. In The fish oocyte: from basic studies to biotechnological applications Edited by: Babin PJ, Cerdà J, Lubzens E. Springer, The Netherlands; 2007:349-396.
59. Carnevali O, Cionna C, Tosti L, Lubzens E, Maradonna F: Role of cathepsins in ovarian follicle growth and maturation. Gen Comp Endocrinol 2006, I46: 195-203.

60. Fabra M, Raldúa D, Power DM, Deen PM, Cerdà J: Marine fish egg hydration is aquaporin-mediated. Science 2005, 307:545.

61. Fabra M, Cerdà J: Ovarian cysteine proteinases in the teleost Fundulus heteroclitus: molecular cloning and gene expression during vitellogenesis and oocyte maturation. Mol Reprod Dev 2004, 67:282-294.

62. Fabra M, Raldúa D, Bozzo MG, Deen PM, Lubzens E, Cerdà J: Yolk proteolysis and aquaporin-l o play essential roles to regulate fish oocyte hydration during meiosis resumption. Dev Biol 2006, 295:250-262.

63. Raldúa D, Fabra M, Bozzo MG, Weber E, Cerdà J: Cathepsin Bmediated yolk protein degradation during killifish oocyte maturation is blocked by an $\mathrm{H}^{+}$-ATPase inhibitor: effects on the hydration mechanism. Am J Physiol Regul Integr Comp Physiol 2006, 290:R456-R466.

64. Gillingham AK, Munro S: The small G proteins of the Arf family and their regulators. Annu Rev Cell Dev Biol 2007, 23:579-6II.

65. Blomhoff R, Blomhoff HK: Overview of retinoid metabolism and function. J Neurobiol 2006, 66:606-630.

66. Ikeda S, Kitagawa M, Imai H, Yamada M: The roles of vitamin A for cytoplasmic maturation of bovine oocytes. J Reprod Dev 2005, 5I:23-35.

67. Wickenheisser JK, Nelson-DeGrave VL, Hendricks KL, Legro RS, Strauss JF 3rd, McAllister JM: Retinoids and retinol differentially regulate steroid biosynthesis in ovarian theca cells isolated from normal cycling women and women with polycystic ovary syndrome. J Clin Endocrinol Metab 2005, 90:4858-65.

68. Levi L, Levavi-Sivan B, Lubzens E: Expression of genes associated with retinoid metabolism in the trout ovarian follicle. Biol Reprod 2008, 79:570-577.

69. DiSepio D, Ghosn C, Eckert RL, Deucher A, Robinson N, Duvic M, Chandraratna RA, Nagpal S: Identification and characterization of a retinoid-induced class II tumor suppressor/growth regulatory gene. Proc Natl Acad Sci USA 1998, 95: I 48I I-| 48 I5.

70. Ireland JLH, Jimenez-Krassel F, Winn ME, Burns DS, Ireland JJ: Evidence for autocrine or paracrine roles of 2-macroglobulin in regulation of estradiol production by granulosa cells and development of dominant follicles. Endocrinology 2004, | 45:2784-2794.

7I. Drummond CD, Bazzoli N, Rizzo E, Sato Y: Postovulatory follicle: a model for experimental studies of programmed cell death or apoptosis in teleosts. J Exp Zool 2000, 287: 176-I82.

72. Thomé RG, Santos HB, Arantes FP, Domingos FF, Bazzoli N, Rizzo E: Dual roles for autophagy during follicular atresia in fish ovary. Autophagy 2009, 5: I 17-II9.

73. Santos HB, Thomé RG, Arantes FP, Sato Y, Bazzoli N, Rizzo E: Ovarian follicular atresia is mediated by heterophagy, autophagy, and apoptosis in Prochilodus argenteus and Leporinus taeniatus (Teleostei: Characiformes). Theriogenology 2008, 70:1449-1460.

74. Santos HB, Sato Y, Moro L, Bazzoli N, Rizzo E: Relationship among follicular apoptosis, integrin betal and collagen type IV during early ovarian regression in the teleost Prochilodus argenteus after induced spawning. Cell Tissue Res 2008, 332:159-170.

75. Lang I: Electron microscopic and histochemical investigations of the atretic oocyte of Perca fluviatilis L. (Teleostei). Cell Tissue Res 198I, 220:20I-2I2.

76. Jong MC, Hofker MH, Havekes LM: Role of ApoCs in lipoprotein metabolism: functional differences between ApoC1, ApoC2, and ApoC3. Arterioscler Thromb Vasc Biol 1999, 19:472-484.

77. Jakobsson $A$, Westerberg $R$, Jacobsson $A$ : Fatty acid elongases in mammals: their regulation and roles in metabolism. Prog Lipid Res 2006, 45:237-249.

78. Babin PJ: Apolipoproteins and the association of egg yolk proteins with plasma high density lipoproteins after ovulation and follicular atresia in the rainbow trout (Salmo gairdneri). J Biol Chem 1987, 262:4290-4296.

79. Agulleiro MJ, André M, Morais S, Cerdà J, Babin PJ: High transcript level of fatty acid-binding protein I I but not of very low-density lipoprotein receptor is correlated to ovarian follicle atresia in a teleost fish (Solea senegalensis). Biol Reprod 2007, 77:504-516. 
80. Wood AW, Kraak G Van Der: Yolk proteolysis in rainbow trout oocytes after serum-free culture: evidence for a novel biochemical mechanism of atresia in oviparous vertebrates. Mol Reprod Dev 2003, 65:219-227.

81. Lauer SJ, Walker D, Elshourbagy NA, Reardon CA, Levy-Wilson B, Taylor JM: Two copies of the human apolipoprotein C-I gene are linked closely to the apolipoprotein E gene. J Biol Chem 1988, 263:7277-7286.

82. MacRae RS, Johnston HM, Mihm M, O'Shaughnessy PJ: Changes in mouse granulosa cell gene expression during early luteinization. Endocrinology 2005, I 46:309-3I7.

83. Zerbinatti CV, Mayer LP, Audet RG, Dyer CA: Apolipoprotein E is a putative autocrine regulator of the rat ovarian theca cell compartment. Biol Reprod 200I, 64:1080-1089.

84. Sarropoulou E, Power DM, Magoulas A, Geisler R, Kotoulas G: Comparative analysis and characterization of expressed sequence tags in gilthead sea bream (Sparus aurata) liver and embryos. Aquaculture 2005, 243:69-8I.

85. Wang Y, Zhou L, Li Z, Gui JF: Molecular cloning and expression characterization of $A p \circ C-I$ in the orange-spotted grouper. Fish Physiol Biochem 2008, 34:339-348.

86. Babin PJ, Thisse C, Durliat M, Andre M, Akimenko MA, Thisse B: Both apolipoprotein $E$ and A-I genes are present in a nonmammalian vertebrate and are highly expressed during embryonic development. Proc Natl Acad Sci USA 1997, 94:8622-8627.

87. Zhou L, Wang Y, Yao B, Li CJ, Ji GD, Gui JF: Molecular cloning and expression pattern of $14 \mathbf{~ k D a}$ apolipoprotein in orange-spotted grouper, Epinephelus coioides. Comp Biochem Physiol B Biochem Mol Biol 2005, I 42:432-437.

88. Huang S, Qiao J, Li R, Wang L, Li M: Can serum apolipoprotein C I demonstrate metabolic abnormality early in women with polycystic ovary syndrome? Fertil Steril 2009. DOI: 10.1016/ j.fertnstert.2009.03.005.

89. Besseau L, Faliex E: Resorption of unemitted gametes in Lithognathus mormyrus (Sparidae, Teleostei): a possible synergic action of somatic and immune cells. Cell Tissue Res 1994 276: $123-132$.

90. Santos HB, Rizzo E, Bazzoli N, Sato Y, Moro L: Ovarian regression and apoptosis in the South American teleost Leportinus taeniatus Lutken (Caraciformes, Anostomidae) from the Sao Francisco Basin. J Fish Biol 2005, 67: | 446-| 459.

91. Townson DH, Liptak AR: Chemokines in the corpus luteum: implications of leukocyte chemotaxis. Reprod Biol Endocrinol 2003, I:94.

92. Yamagoe S, Yamakawa Y, Matsuo Y, Minowada J, Mizuno S, Suzuki K Purification and primary amino acid sequence of a novel neutrophil chemotactic factor LECT2. Immunol Lett 1996, 52:9-I.

93. Donato R: SI00: a multigenic family of calcium-modulated proteins of the EF-hand type with intracellular and extracellular functional roles. Int / Biochem Cell Biol 200I, 33:637-668.

94. Chun SY, Bae HW, Kim WJ, Park JH, Hsu SY, Hsueh AJ: Expression of messenger ribonucleic acid for the antiapoptosis gene $\mathrm{PII}$ in the rat ovary: gonadotropin stimulation in granulosa cells of preovulatory follicles. Endocrinology 200I, I 42:23II-23 I7.

95. Bobe J, Goetz FW: A SI00 homologue mRNA isolated by differential display PCR is down-regulated in the brook trout (Salvelinus fontinalis) post-ovulatory ovary. Gene 2000, 257: 187-194.

96. Thomas FH, Wilson H, Silvestri A, Fraser HM: Thrombospondin-I expression is increased during follicular atresia in the primate ovary. Endocrinology 2008, 149:185-192.

97. Petrik JJ, Gentry PA, Feige JJ, LaMarre J: Expression and localization of thrombospondin- $I$ and -2 and their cell-surface receptor, CD36, during rat follicular development and formation of the corpus luteum. Biol Reprod 2002, 67:|522-I56|.

98. Schroeder A, Mueller O, Stocker S, Salowsky R, Leiber M, Gassmann M, Lightfoot S, Menzel W, Granzow M, Ragg T: The RIN: an RNA integrity number for assigning integrity values to RNA measurements. BMC Mol Biol 2006, 7:3.

99. Van Gelder RN, von Zastrow ME, Yool A, Dement WC, Barchas JD, Eberwine $\mathrm{JH}$ : Amplified RNA synthesized from limited quantities of heterogeneous cDNA. Proc Natl Acad Sci USA 1990, 87:1663-1667.

100. Workman C, Jensen LJ, Jarmer H, Berka R, Gautier L, Nielsen HB, Saxild $\mathrm{H}-\mathrm{H}$, Nielsen $\mathrm{C}$, Brunak S, Knudsen S: A new non-linear nor- malization method for reducing variability in DNA microarray experiments. Genome Biol 2002, 3:research0048.

101. Baldi P, Long AD: A Bayesian framework for the analysis of microarray expression data: regularized $t$-test and statistical inferences of gene changes. Bioinformatics 200I, 17:509-519.

102. National Center for Biotechnology Information (NCBI) [http://blast.ncbi.nlm.nih.gov/Blast.cgi]

103. Edgar R, Domrachev M, Lash AE: Gene Expression Omnibus: NCBI gene expression and hybridization array data repository. Nucleic Acids Res 2002, 30:207-2 I0.

104. National Center for Biotechnology Information (NCBI). Gene Expression Omnibus [http://www.ncbi.nlm.nih.gov/ projects/geo/query/acc.cgi?acc=GSE 17337]

105. De Castro E, Sigrist CJA, Gattiker A, Bulliard V, Langendijk-Genevaux PS, Gasteiger E, Bairoch A, Hulo N: ScanProsite: detection of PROSITE signature matches and ProRule-associated functional and structural residues in proteins. Nucleic Acids Res 2006, 34:W362-W365.

106. Higgins D, Thompson J, Gibson T, Thompson JD, Higgins DG, Gibson T]: CLUSTAL W: improving the sensitivity of progressivemultiple sequence alignment through sequence weighting, position-specific gap penalties and weight matrix choice. Nucleic Acids Res 1994, 22:4673-4680.

107. Saitou N, Nei M: The neighbor-joining method: a new method for reconstructing phylogenetic trees. Mol Biol Evol 1987, 4:406-425.

108. Felsenstein J: Confidence limits on phylogenies: an approach suing the bootstrap. Evolution 1985, 39:783-791.

109. Cerdà J, Chauvigne F, Agulleiro MJ, Marin E, Halm S, MartínezRodríguez G, Prat F: Molecular cloning of Senegalese sole (Solea senegalensis) follicle-stimulating hormone and luteinizing hormone subunits and expression pattern during spermatogenesis. Gen Comp Endocrinol 2008, 156:470-48I.

Publish with Bio Med Central and every scientist can read your work free of charge

"BioMed Central will be the most significant development for disseminating the results of biomedical research in our lifetime. "

Sir Paul Nurse, Cancer Research UK

Your research papers will be:

- available free of charge to the entire biomedical community

- peer reviewed and published immediately upon acceptance

- cited in PubMed and archived on PubMed Central

- yours - you keep the copyright
BioMedcentral 